\title{
Some new spaces of Besov and Triebel-Lizorkin type on homogeneous spaces
}

\author{
by \\ Yongsheng Han (Auburn, AL) and Dachun Yang (Beijing)
}

\begin{abstract}
New norms for some distributions on spaces of homogeneous type which include some fractals are introduced. Using inhomogeneous discrete Calderón reproducing formulae and the Plancherel-Pólya inequalities on spaces of homogeneous type, the authors prove that these norms give a new characterization for the Besov and TriebelLizorkin spaces with $p, q>1$ and can be used to introduce new inhomogeneous Besov and Triebel-Lizorkin spaces with $p, q \leq 1$ on spaces of homogeneous type. Moreover, atomic decompositions of these new spaces are also obtained. All the results of this paper are new even for $\mathbb{R}^{n}$.
\end{abstract}

1. Introduction. Spaces of homogeneous type introduced by Coifman and Weiss in [2] include the Euclidean space, the $n$-torus in $\mathbb{R}^{n}$, the $C^{\infty}$ compact Riemannian manifolds, the boundaries of Lipschitz domains and, in particular, the Lipschitz manifolds introduced recently by Triebel in [19] and the $d$-sets in $\mathbb{R}^{n}$. It has been proved by Triebel in [17] that the $d$-sets in $\mathbb{R}^{n}$ include various kinds of fractals; see also [18].

The homogeneous Besov and Triebel-Lizorkin spaces on spaces of homogeneous type have been studied in [12] and [7]. In [9], the inhomogeneous Besov and Triebel-Lizorkin spaces on spaces of homogeneous type were introduced by use of the generalized Littlewood-Paley $g$-functions when $p, q \geq 1$. In [10], the inhomogeneous Triebel-Lizorkin spaces were generalized to the cases where $0<p_{0}<p \leq 1 \leq q<\infty$ via the generalized Littlewood-Paley $S$-functions. The main purpose of this paper is to generalize the inhomogeneous Besov and Triebel-Lizorkin spaces on spaces of homogeneous type to the case where $p, q \leq 1$. To do this, we first introduce

2000 Mathematics Subject Classification: Primary 42B35; Secondary 42B25, 46E35, 43A99.

Key words and phrases: space of homogeneous type, Plancherel-Pólya inequality, Besov space, Triebel-Lizorkin space, Calderón reproducing formula, Littlewood-Paley $S$ function, Littlewood-Paley $g$-function, unit, molecule.

The second (corresponding) author gratefully acknowledges the support of the Alexander von Humboldt Foundation of Germany, NNSF (No. 10271015) and RFDP (No. 20020027004) of China. 
new norms for distributions on spaces of homogeneous type. Using the inhomogeneous Calderón reproducing formulas ([11]) and the Plancherel-Pólya inequalities ([4]) on spaces of homogeneous type, we prove that these new norms are equivalent to those introduced in [9]. Thus, these norms give a new characterization for the inhomogeneous Besov and Triebel-Lizorkin spaces developed in [9]. Moreover, using these norms, we introduce new inhomogeneous Besov and Triebel-Lizorkin spaces for the case where $p, q \leq 1$. We also introduce a new inhomogeneous generalized Littlewood-Paley $g$ function on spaces of homogeneous type and show that the $L^{p}(X)$ norms of this $g$-function and of the inhomogeneous Littlewood-Paley $S$-function introduced in [10] are equivalent. As a consequence, this gives a new characterization of Triebel-Lizorkin spaces ([10]). Finally, atomic decompositions of these spaces are obtained. As a simple application, one can easily see that when one regards a Lipschitz manifold as in [19] as a space of homogeneous type, the Besov and Triebel-Lizorkin spaces defined here are the same as those defined by Triebel in [19] via a completely different approach. More applications of these new Besov and Triebel-Lizorkin spaces will be given elsewhere; see [13] and [20]. All results of this paper are new even for $\mathbb{R}^{n}$.

The organization of this paper is as follows. We state all the main results in the remaining part of this section, and all the proofs will be given in Section 2. Some remarks will be given in Section 3.

Before stating the main results, we first recall the necessary definitions and notation for spaces of homogeneous type.

A quasi-metric $\varrho$ on a set $X$ is a function $\varrho: X \times X \rightarrow[0, \infty)$ satisfying

(i) $\varrho(x, y)=0$ if and only if $x=y$;

(ii) $\varrho(x, y)=\varrho(y, x)$ for all $x, y \in X$;

(iii) there exists a constant $A \in[1, \infty)$ such that for all $x, y, z \in X$,

$$
\varrho(x, y) \leq A[\varrho(x, z)+\varrho(z, y)] .
$$

Any quasi-metric defines a topology, for which the balls

$$
B(x, r)=\{y \in X: \varrho(y, x)<r\}
$$

for all $x \in X$ and all $r>0$ form a basis.

In what follows, we set $\operatorname{diam} X=\sup \{\varrho(x, y): x, y \in X\}$. We also make the following conventions. We write $f \sim g$ if there is a constant $C>0$ independent of the main parameters such that $C^{-1} g<f<C g$. Throughout the paper, we will denote by $C$ a positive constant which is independent of the main parameters, but it may vary from line to line. Constants with subscripts, such as $C_{1}$, do not change in different occurrences. We denote $\mathbb{N} \cup\{0\}$ simply by $\mathbb{Z}_{+}$and for any $q \in[1, \infty]$, we denote by $q^{\prime}$ its conjugate index, namely, $1 / q+1 / q^{\prime}=1$. If $A$ is a set, we denote by $\chi_{A}$ the characteristic function of $A$. 
Definition 1 ([13]). Let $d>0$ and $0<\theta \leq 1$. A space of homogeneous type, $(X, \varrho, \mu)_{d, \theta}$, is a set $X$ together with a quasi-metric $\varrho$ and a nonnegative Borel regular measure $\mu$ on $X$ such that $\operatorname{supp} \mu=X$ and there exists a constant $C_{0}>0$ such that for all $0<r<\operatorname{diam} X$ and all $x, x^{\prime}, y \in X$,

$$
\begin{gathered}
\mu(B(x, r)) \sim r^{d} \\
\left|\varrho(x, y)-\varrho\left(x^{\prime}, y\right)\right| \leq C_{0} \varrho\left(x, x^{\prime}\right)^{\theta}\left[\varrho(x, y)+\varrho\left(x^{\prime}, y\right)\right]^{1-\theta} .
\end{gathered}
$$

A space of homogeneous type defined above is a variant of a space of homogeneous type introduced by Coifman and Weiss in [2]. In [14], Macías and Segovia have proved that one can replace the quasi-metric $\varrho$ of any space of homogeneous type in the sense of Coifman and Weiss by another quasimetric $\bar{\varrho}$ which yields the same topology and $(X, \bar{\varrho}, \mu)$ is as in Definition 1 with $d=1$.

Definition 2 ([8]). Fix $\gamma>0$ and $\theta \geq \beta>0$. A function $f$ defined on $X$ is said to be a test function of type $\left(x_{0}, r, \beta, \gamma\right)$ with $x_{0} \in X$ and $r>0$ if $f$ satisfies the following conditions:

(i) $|f(x)| \leq C \frac{r^{\gamma}}{\left(r+\varrho\left(x, x_{0}\right)\right)^{d+\gamma}}$;

(ii) $|f(x)-f(y)| \leq C\left(\frac{\varrho(x, y)}{r+\varrho\left(x, x_{0}\right)}\right)^{\beta} \frac{r^{\gamma}}{\left(r+\varrho\left(x, x_{0}\right)\right)^{d+\gamma}}$

$$
\text { for } \varrho(x, y) \leq \frac{1}{2 A}\left[r+\varrho\left(x, x_{0}\right)\right] \text {. }
$$

If $f$ is a test function of type $\left(x_{0}, r, \beta, \gamma\right)$, we write $f \in \mathcal{G}\left(x_{0}, r, \beta, \gamma\right)$, and the norm of $f$ in $\mathcal{G}\left(x_{0}, r, \beta, \gamma\right)$ is defined by $\|f\|_{\mathcal{G}\left(x_{0}, r, \beta, \gamma\right)}=\inf \{C$ : (i) and (ii) hold\}.

Now fix $x_{0} \in X$ and let $\mathcal{G}(\beta, \gamma)=\mathcal{G}\left(x_{0}, 1, \beta, \gamma\right)$. It is easy to see that

$$
\mathcal{G}\left(x_{1}, r, \beta, \gamma\right)=\mathcal{G}(\beta, \gamma)
$$

with equivalent norms for all $x_{1} \in X$ and $r>0$. Furthermore, it is easy to check that $\mathcal{G}(\beta, \gamma)$ is a Banach space. Also, let $(\mathcal{G}(\beta, \gamma))^{\prime}$ be the dual space of all continuous linear functionals on $\mathcal{G}(\beta, \gamma)$.

We denote by $\langle h, f\rangle$ the natural pairing of $h \in(\mathcal{G}(\beta, \gamma))^{\prime}$ and $f \in \mathcal{G}(\beta, \gamma)$. Clearly, for all $h \in(\mathcal{G}(\beta, \gamma))^{\prime},\langle h, f\rangle$ is well defined for all $f \in \mathcal{G}\left(x_{0}, r, \beta, \gamma\right)$ with $x_{0} \in X$ and $r>0$.

It is well known that even when $X=\mathbb{R}^{n}, \mathcal{G}\left(\beta_{1}, \gamma\right)$ is not dense in $\mathcal{G}\left(\beta_{2}, \gamma\right)$ if $\beta_{1}>\beta_{2}$, which will bring us some inconvenience. To overcome this defect, in what follows, we let $\stackrel{\circ}{\mathcal{G}}(\beta, \gamma)$ be the completion of the space $\mathcal{G}(\theta, \theta)$ in $\mathcal{G}(\beta, \gamma)$ when $0<\beta, \gamma<\theta$. (The authors thank Professor Hans Triebel for suggesting this idea.) 
Definition $3([8])$. A sequence $\left\{S_{k}\right\}_{k=0}^{\infty}$ of linear operators is said to be an approximation to the identity of order $\varepsilon \in(0, \theta]$ if there exists $C_{1}>0$ such that for all $k \in \mathbb{Z}_{+}$and all $x, x^{\prime}, y, y^{\prime} \in X$, the kernel $S_{k}(x, y)$ of $S_{k}$ is a function from $X \times X$ into $\mathbb{C}$ satisfying

(i) $\left|S_{k}(x, y)\right| \leq C_{1} \frac{2^{-k \varepsilon}}{\left(2^{-k}+\varrho(x, y)\right)^{d+\varepsilon}}$;

(ii) $\left|S_{k}(x, y)-S_{k}\left(x^{\prime}, y\right)\right| \leq C_{1}\left(\frac{\varrho\left(x, x^{\prime}\right)}{2^{-k}+\varrho(x, y)}\right)^{\varepsilon} \frac{2^{-k \varepsilon}}{\left(2^{-k}+\varrho(x, y)\right)^{d+\varepsilon}}$ for $\varrho\left(x, x^{\prime}\right) \leq \frac{1}{2 A}\left(2^{-k}+\varrho(x, y)\right)$;

(iii) $\left|S_{k}(x, y)-S_{k}\left(x, y^{\prime}\right)\right| \leq C_{1}\left(\frac{\varrho\left(y, y^{\prime}\right)}{2^{-k}+\varrho(x, y)}\right)^{\varepsilon} \frac{2^{-k \varepsilon}}{\left(2^{-k}+\varrho(x, y)\right)^{d+\varepsilon}}$ for $\varrho\left(y, y^{\prime}\right) \leq \frac{1}{2 A}\left(2^{-k}+\varrho(x, y)\right)$;

(iv) $\left|\left[S_{k}(x, y)-S_{k}\left(x, y^{\prime}\right)\right]-\left[S_{k}\left(x^{\prime}, y\right)-S_{k}\left(x^{\prime}, y^{\prime}\right)\right]\right|$ $\leq C_{1}\left(\frac{\varrho\left(x, x^{\prime}\right)}{2^{-k}+\varrho(x, y)}\right)^{\varepsilon}\left(\frac{\varrho\left(y, y^{\prime}\right)}{2^{-k}+\varrho(x, y)}\right)^{\varepsilon} \frac{2^{-k \varepsilon}}{\left(2^{-k}+\varrho(x, y)\right)^{d+\varepsilon}}$ for $\varrho\left(x, x^{\prime}\right) \leq \frac{1}{2 A}\left(2^{-k}+\varrho(x, y)\right)$ and $\varrho\left(y, y^{\prime}\right) \leq \frac{1}{2 A}\left(2^{-k}+\varrho(x, y)\right)$;

(v) $\int_{X} S_{k}(x, y) d \mu(y)=1$;

(vi) $\int_{X} S_{k}(x, y) d \mu(x)=1$.

REMARK 1. Coifman's construction in [3] yields an approximation to the identity of order $\theta$ such that $S_{k}(x, y)$ has a compact support when one variable is fixed, namely, there is a constant $C_{2}>0$ such that for all $k \in \mathbb{Z}_{+}$, $S_{k}(x, y)=0$ if $\varrho(x, y) \geq C_{2} 2^{-k}$.

We need the following construction of Christ [1], which provides an analogue of the grid of Euclidean dyadic cubes on spaces of homogeneous type.

Lemma 1. Let $X$ be a space of homogeneous type. Then there exist a collection

$$
\left\{Q_{\alpha}^{k} \subset X: k \in \mathbb{Z}_{+}, \alpha \in I_{k}\right\}
$$

of open subsets, where $I_{k}$ is some (possibly finite) index set, and constants $\delta \in(0,1)$ and $C_{3}, C_{4}>0$ such that

(i) $\mu\left(X \backslash \bigcup_{\alpha} Q_{\alpha}^{k}\right)=0$ for each fixed $k$ and $Q_{\alpha}^{k} \cap Q_{\beta}^{k}=\emptyset$ if $\alpha \neq \beta$;

(ii) for any $\alpha, \beta, k, l$ with $l \geq k$, either $Q_{\beta}^{l} \subset Q_{\alpha}^{k}$ or $Q_{\beta}^{l} \cap Q_{\alpha}^{k}=\emptyset$;

(iii) for each $(k, \alpha)$ and each $l<k$ there is a unique $\beta$ such that $Q_{\alpha}^{k} \subset Q_{\beta}^{l}$; 
(iv) $\operatorname{diam}\left(Q_{\alpha}^{k}\right) \leq C_{3} \delta^{k}$;

(v) each $Q_{\alpha}^{k}$ contains some ball $B\left(z_{\alpha}^{k}, C_{4} \delta^{k}\right)$, where $z_{\alpha}^{k} \in X$.

In fact, we can think of $Q_{\alpha}^{k}$ as being a dyadic cube with diameter roughly $\delta^{k}$ and centered at $z_{\alpha}^{k}$. In what follows, we always suppose $\delta=1 / 2$. See [12] for how to remove this restriction. Also, in the following, for $k \in \mathbb{Z}_{+}$and $\tau \in I_{k}$, we will denote by $Q_{\tau}^{k, \nu}, \nu=1, \ldots, N(k, \tau)$, the set of all cubes $Q_{\tau^{\prime}}^{k+j} \subset Q_{\tau}^{k}$, where $j$ is a fixed large positive integer. Denote by $y_{\tau}^{k, \nu}$ a point in $Q_{\tau}^{k, \nu}$. For another fixed large positive integer $\bar{j}, k \in \mathbb{Z}_{+}$and $\tau \in I_{k}$, we will denote by $\bar{Q}_{\tau}^{k, \nu}, \nu=1, \ldots, \bar{N}(k, \tau)$, the set of all cubes $Q_{\tau^{\prime}}^{k+\bar{j}} \subset Q_{\tau}^{k}$ and by $\bar{y}_{\tau}^{k, \nu}$ a point in $\bar{Q}_{\tau}^{k, \nu}$. For any dyadic cube $Q$ and any $f \in L_{\text {loc }}^{1}(X)$, we set

$$
m_{Q}(f)=\frac{1}{\mu(Q)} \int_{Q} f(x) d \mu(x) .
$$

Now we introduce $B_{p q}^{s}(X)$ and $F_{p q}^{s}(X)$ norms for all $f \in(\stackrel{\circ}{\mathcal{G}}(\beta, \gamma))^{\prime}$ with $0<\beta, \gamma<\theta$.

Definition 4. Let $-\theta<s<\theta$. Suppose that $\left\{S_{k}\right\}_{k=0}^{\infty}$ is an approximation to the identity of order $\theta, E_{k}=S_{k}-S_{k-1}$ for $k \in \mathbb{N}, E_{0}=S_{0}$ and $\left\{Q_{\tau}^{0, \nu}: \tau \in I_{0}, \nu=1, \ldots, N(0, \tau)\right\}$ with a fixed large $j$ are dyadic cubes as above. For $0<p, q \leq \infty$ and all $f \in(\stackrel{\mathcal{G}}{ }(\beta, \gamma))^{\prime}$ with $|s|<\beta<\theta$ and $0<\gamma<\theta$, define

$$
\begin{aligned}
\|f\|_{B_{p q}^{s}(X)}= & \left\{\sum_{\tau \in I_{0}} \sum_{\nu=1}^{N(0, \tau)} \mu\left(Q_{\tau}^{0, \nu}\right)\left[m_{Q_{\tau}^{0, \nu}}\left(\left|E_{0}(f)\right|\right)\right]^{p}\right\}^{1 / p} \\
& +\left\{\sum_{k=1}^{\infty}\left[2^{k s}\left\|E_{k}(f)\right\|_{L^{p}(X)}\right]^{q}\right\}^{1 / q}
\end{aligned}
$$

and for $0<p<\infty, 0<q \leq \infty$,

$$
\begin{aligned}
\|f\|_{F_{p q}^{s}(X)}= & \left\{\sum_{\tau \in I_{0}} \sum_{\nu=1}^{N(0, \tau)} \mu\left(Q_{\tau}^{0, \nu}\right)\left[m_{Q_{\tau}^{0, \nu}}\left(\left|E_{0}(f)\right|\right)\right]^{p}\right\}^{1 / p} \\
& +\left\|\left\{\sum_{k=1}^{\infty}\left[2^{k s}\left|E_{k}(f)\right|\right]^{q}\right\}^{1 / q}\right\|_{L^{p}(X)} .
\end{aligned}
$$

Here, for $k \in \mathbb{Z}_{+}$and any suitable $f$,

$$
E_{k}(f)(x)=\int_{X} E_{k}(x, y) f(y) d \mu(y) .
$$

We now introduce new inhomogeneous generalized Littlewood-Paley $g$ functions. 
Definition 5. Let $s \in(-\theta, \theta)$ and $q \in(0, \infty)$. Let $\left\{E_{k}\right\}_{k=0}^{\infty}$ and

$$
\left\{Q_{\tau}^{0, \nu}: \tau \in I_{0}, \nu=1, \ldots, N(0, \tau)\right\}
$$

with a fixed large $j$ be as in Definition 4. The inhomogeneous generalized Littlewood-Paley g-function is defined by

$$
g_{q}^{s}(f)(x)=\sum_{\tau \in I_{0}} \sum_{\nu=1}^{N(0, \tau)}\left[m_{Q_{\tau}^{0, \nu}}\left(\left|E_{0}(f)\right|\right)\right] \chi_{Q_{\tau}^{0, \nu}}(x)+\left\{\sum_{k=1}^{\infty}\left[2^{k s}\left|E_{k}(f)(x)\right|\right]^{q}\right\}^{1 / q}
$$

for $f \in(\stackrel{\circ}{\mathcal{G}}(\beta, \gamma))^{\prime}$ with $|s|<\beta<\theta$ and $0<\gamma<\theta$.

Let us now state the main theorems of this paper.

Theorem 1. Let $s \in(-\theta, \theta)$. Let $\left\{S_{k}\right\}_{k=0}^{\infty}$ and $\left\{P_{k}\right\}_{k=0}^{\infty}$ be two approximations to the identity of order $\theta$ as in Definition $3, E_{k}=S_{k}-S_{k-1}$ and $D_{k}=P_{k}-P_{k-1}$ for $k \in \mathbb{N}$, and $E_{0}=S_{0}$ and $D_{0}=P_{0}$. Then, if $\max (d /(d+\theta), d /(d+\theta+s))<p \leq \infty$ and $0<q \leq \infty$, for all $f \in(\stackrel{\mathcal{G}}{ }(\beta, \gamma))^{\prime}$ with $|s|<\beta<\theta$ and $0<\gamma<\theta$, we have

$$
\begin{aligned}
& \left\{\sum_{\tau \in I_{0}} \sum_{\nu=1}^{N(0, \tau)} \mu\left(Q_{\tau}^{0, \nu}\right)\left[m_{Q_{\tau}^{0, \nu}}\left(\left|E_{0}(f)\right|\right)\right]^{p}\right\}^{1 / p}+\left\{\sum_{k=1}^{\infty}\left[2^{k s}\left\|E_{k}(f)\right\|_{L^{p}(X)}\right]^{q}\right\}^{1 / q} \\
& \sim\left\{\sum_{\tau \in I_{0}} \sum_{\nu=1}^{N(0, \tau)} \mu\left(Q_{\tau}^{0, \nu}\right)\left[m_{Q_{\tau}^{0, \nu}}\left(\left|D_{0}(f)\right|\right)\right]^{p}\right\}^{1 / p}+\left\{\sum_{k=1}^{\infty}\left[2^{k s}\left\|D_{k}(f)\right\|_{L^{p}(X)}\right]^{q}\right\}^{1 / q} .
\end{aligned}
$$

If $\max (d /(d+\theta), d /(d+\theta+s))<p, q$ and $p<\infty, q \leq \infty$, then for all $f \in(\stackrel{\circ}{\mathcal{G}}(\beta, \gamma))^{\prime}$ with $|s|<\beta<\theta$ and $0<\gamma<\theta$, we have

$$
\begin{aligned}
\left\|g_{q}^{s}(f)\right\|_{L^{p}(X)} \sim & \left\{\sum_{\tau \in I_{0}} \sum_{\nu=1}^{N(0, \tau)} \mu\left(Q_{\tau}^{0, \nu}\right)\left[m_{Q_{\tau}^{0, \nu}}\left(\left|D_{0}(f)\right|\right)\right]^{p}\right\}^{1 / p} \\
& +\left\|\left\{\sum_{k=1}^{\infty}\left[2^{k s}\left|D_{k}(f)\right|\right]^{q}\right\}^{1 / q}\right\|_{L^{p}(X)} \cdot
\end{aligned}
$$

Theorem 1 shows that Definition 4 is independent of the choices of large positive integers $j$ and approximations to the identity. The following theorem shows that the norms defined by Definition 4 give new characterizations for the Besov spaces $B_{p q}^{s}(X)$ and Triebel-Lizorkin spaces $F_{p q}^{s}(X)$ developed in [9] and [10].

Theorem 2. Let $s \in(-\theta, \theta)$ and $\left\{E_{k}\right\}_{k=0}^{\infty}$ be as in Theorem 1. Then, for all $f \in(\stackrel{\circ}{\mathcal{G}}(\beta, \gamma))^{\prime}$ with $|s|<\beta<\theta$ and $0<\gamma<\theta$, 


$$
\begin{aligned}
\left\{\sum_{\tau \in I_{0}} \sum_{\nu=1}^{N(0, \tau)} \mu\left(Q_{\tau}^{0, \nu}\right)\left[m_{Q_{\tau}^{0, \nu}}\left(\left|E_{0}(f)\right|\right)\right]^{p}\right\}^{1 / p} & +\left\{\sum_{k=1}^{\infty}\left[2^{k s}\left\|E_{k}(f)\right\|_{L^{p}(X)}\right]^{q}\right\}^{1 / q} \\
& \sim\left\{\sum_{k=0}^{\infty}\left[2^{k s}\left\|E_{k}(f)\right\|_{L^{p}(X)}\right]^{q}\right\}^{1 / q}
\end{aligned}
$$

when $1 \leq p, q \leq \infty$; while when $1 \leq p<\infty$ and $1 \leq q \leq \infty$,

$$
\left\|g_{q}^{s}(f)\right\|_{L^{p}(X)} \sim\left\|\left\{\sum_{k=0}^{\infty}\left[2^{k s}\left|E_{k}(f)\right|\right]^{q}\right\}^{1 / q}\right\|_{L^{p}(X)} .
$$

If $\max (d /(d+\theta), d /(d+\theta+s))<p<\infty$ and $1 \leq q<\infty$, then for all $f \in(\stackrel{\mathcal{G}}{(} \beta, \gamma))^{\prime}$ with $|s|<\beta<\theta$ and $0<\gamma<\theta$,

$$
\left\|S_{q, a}^{s}(f)\right\|_{L^{p}(X)} \sim\left\|g_{q}^{s}(f)\right\|_{L^{p}(X)},
$$

where $a \in(0, \infty)$ and the generalized Littlewood-Paley function $S_{q, a}^{s}(f)$ is defined by

$$
S_{q, a}^{s}(f)(x)=\left\{\sum_{k=0}^{\infty} \int_{\left\{y: \varrho(x, y) \leq a 2^{-k}\right\}} 2^{k d}\left[2^{k s}\left|E_{k}(f)(y)\right|\right]^{q} d \mu(y)\right\}^{1 / q} .
$$

The following theorem shows that the norms given by Definition 4 are also independent of the choices of the distribution spaces $(\mathcal{G}(\beta, \gamma))^{\prime}$. In what follows, we let $a_{+}=\max (a, 0)$ for any $a \in \mathbb{R}$.

Theorem 3. Let $s \in(-\theta, \theta)$.

(i) If $\max (d /(d+\theta), d /(d+\theta+s))<p \leq \infty, 0<q \leq \infty, f \in\left(\stackrel{\circ}{\mathcal{G}}\left(\beta_{1}, \gamma_{1}\right)\right)^{\prime}$ with $\max \left(0, s_{+},-s+d(1 / p-1)_{+}\right)<\beta_{1}<\theta, 0<\gamma_{1}<\theta$ and $\|f\|_{B_{p q}^{s}(X)}<\infty$, then $f \in\left(\stackrel{\mathcal{G}}{(}\left(\beta_{2}, \gamma_{2}\right)\right)^{\prime}$ with $\max \left(0, s_{+},-s+d(1 / p-1)_{+}\right)<\beta_{2}<\beta_{1}$ and $0<\gamma_{2}<\gamma_{1}$.

(ii) If $\max (d /(d+\theta), d /(d+\theta+s))<p, q$ and $p<\infty, q \leq \infty, f \in$ $\left(\stackrel{\mathcal{G}}{(}\left(\beta_{1}, \gamma_{1}\right)\right)^{\prime}$ with $\max \left(0, s_{+},-s+d(1 / p-1)_{+}\right)<\beta_{1}<\theta, 0<\gamma_{1}<\theta$ and $\|f\|_{F_{p q}^{s}(X)}<\infty$, then $f \in\left(\stackrel{\mathcal{G}}{ }\left(\beta_{2}, \gamma_{2}\right)\right)^{\prime}$ with $\max \left(0, s_{+},-s+d(1 / p-1)_{+}\right)<$ $\beta_{2}<\beta_{1}$ and $0<\gamma_{2}<\gamma_{1}$.

We are now ready to introduce the spaces $B_{p q}^{s}(X)$ and $F_{p q}^{s}(X)$ with $p, q \leq 1$.

Definition 6. Let $s \in(-\theta, \theta)$ and $\left\{E_{k}\right\}_{k=0}^{\infty}$ be as in Definition 4.

(i) The inhomogeneous Besov space $B_{p q}^{s}(X)$ for $\max (d /(d+\theta)$, $d /(d+\theta+s))<p \leq \infty$ and $0<q \leq \infty$ is the collection of all $f \in(\stackrel{\circ}{\mathcal{G}}(\beta, \gamma))^{\prime}$ with any given $\beta$ and $\gamma$ satisfying $\max \left(0, s_{+},-s+d(1 / p-1)_{+}\right)<\beta<\theta$ and $d(1 / p-1)_{+}<\gamma<\theta$ such that $\|f\|_{B_{p q}^{s}(X)}<\infty$. 
(ii) The inhomogeneous Triebel-Lizorkin space $F_{p q}^{s}(X)$ for $\max (d /(d+\theta)$, $d /(d+\theta+s))<p<\infty$ and $\max (d /(d+\theta), d /(d+\theta+s))<q \leq \infty$ is the collection of all $f \in(\stackrel{\mathcal{G}}{(\beta, \gamma}, \gamma))^{\prime}$ with any given $\beta$ and $\gamma$ satisfying $\max \left(0, s_{+},-s+d(1 / p-1)_{+}\right)<\beta<\theta$ and $d(1 / p-1)_{+}<\gamma<\theta$ such that $\|f\|_{F_{p q}^{s}(X)}<\infty$.

REMARK 2. The restrictions $s_{+}<\beta \leq \theta$ and $d(1 / p-1)_{+}<\gamma \leq \theta$ guarantee that $\mathcal{G}(\beta, \gamma) \subset B_{p q}^{s}(X) \cap F_{p q}^{s}(X)$ for $s, p$ and $q$ as in Definition 6 (see Lemma 2.1 in [21] for the proof); while the restrictions $\max (0,-s+$ $\left.d(1 / p-1)_{+}\right)<\beta<\theta$ and $0<\gamma<\theta$ guarantee that the definitions of the spaces $B_{p q}^{s}(X)$ and $F_{p q}^{s}(X)$ are independent of the choices of $\beta$ and $\gamma$ satisfying these conditions by Theorem 3. Thus, if $\beta$ and $\gamma$ are as in Definition 6, then

$$
\stackrel{\circ}{\mathcal{G}}(\beta, \gamma) \subset \mathcal{G}(\beta, \gamma) \subset B_{p q}^{s}(X) \cap F_{p q}^{s}(X), \quad B_{p q}^{s}(X) \cup F_{p q}^{s}(X) \subset(\stackrel{\circ}{\mathcal{G}}(\beta, \gamma))^{\prime},
$$

for $s, p$ and $q$ as in Definition 6.

To give atomic characterizations of the spaces $B_{p q}^{s}(X)$ and $F_{p q}^{s}(X)$, as in the case $X=\mathbb{R}^{n}$ (see [6]), we need certain inhomogeneous spaces of sequences indexed by "dyadic cubes" $\left\{Q_{\tau}^{k, \nu}: k \in \mathbb{Z}_{+}, \tau \in I_{k}, \nu=1, \ldots, N(k, \tau)\right\}$ $\equiv \mathbb{J}$ in $X$, which will characterize the coefficients in the decompositions of distributions in $B_{p q}^{s}(X)$ and $F_{p q}^{s}(X)$ in terms of smooth atoms, blocks, molecules and units. For $-\theta<s<\theta$ and $0<p, q<\infty$, we let $b_{p q}^{s}(X)$ be the collection of sequences $\lambda=\left\{\lambda_{Q}\right\}_{Q \in \mathbb{J}}$ such that

$$
\|\lambda\|_{b_{p q}^{s}(X)}=\left\{\sum_{k=0}^{\infty}\left[\sum_{\tau \in I_{k}} \sum_{\nu=1}^{N(k, \tau)} 2^{k s p}\left(\mu\left(Q_{\tau}^{k, \nu}\right)^{1 / p-1 / 2}\left|\lambda_{Q_{\tau}^{k, \nu}}\right|\right)^{p}\right]^{q / p}\right\}^{1 / q}<\infty .
$$

Let $f_{p q}^{s}(X)$ be the collection of sequences $\lambda=\left\{\lambda_{Q}\right\}_{Q \in \mathbb{J}}$ such that

$$
\|\lambda\|_{f_{p q}^{s}(X)}=\left\|\left\{\sum_{k=0}^{\infty} \sum_{\tau \in I_{k}} \sum_{\nu=0}^{N(k, \tau)}\left(2^{k s} \mu\left(Q_{\tau}^{k, \nu}\right)^{-1 / 2}\left|\lambda_{Q_{\tau}^{k, \nu}}\right| \chi_{Q_{\tau}^{k, \nu}}\right)^{q}\right\}^{1 / q}\right\|_{L^{p}(X)}<\infty .
$$

The following smooth blocks and atoms were introduced in [9].

Definition 7. Fix $j \in \mathbb{N}$ and a collection of open subsets,

$$
\left\{Q_{\tau}^{k, \nu}: k \in \mathbb{Z}_{+}, \tau \in I_{k}, \nu=1, \ldots, N(k, \tau)\right\},
$$

satisfying the conditions as above. A function $a_{Q_{\tau}^{k, \nu}}$ defined on $X$ is said to be a $\gamma$-smooth atom for $Q_{\tau}^{k, \nu}$ if

(i) $\operatorname{supp} a_{Q_{\tau}^{k, \nu}} \subset B\left(y_{\tau}^{k, \nu}, 3 A C_{2} 2^{-k}\right)$, where $y_{\tau}^{k, \nu}$ is the center of $Q_{\tau}^{k, \nu}$;

(ii) $\int_{X} a_{Q_{\tau}^{k}}(x) d \mu(x)=0$;

(iii) $\left|a_{Q_{\tau}^{k, \nu}}(x)\right| \leq \mu\left(Q_{\tau}^{k, \nu}\right)^{-1 / 2}$ and

$$
\left|a_{Q_{\tau}^{k, \nu}}(x)-a_{Q_{\tau}^{k, \nu}}(y)\right| \leq \mu\left(Q_{\tau}^{k, \nu}\right)^{-1 / 2-\gamma / d} \varrho(x, y)^{\gamma} .
$$


A function $a_{Q_{\tau}^{k, \nu}}$ defined on $X$ is said to be a $\gamma$-smooth block for $Q_{\tau}^{k, \nu}$ if $a_{Q_{\tau}^{k, \nu}}$ satisfies only (i) and (iii) above.

TheOREM 4. Let $s \in(-\theta, \theta), j \in \mathbb{N}$ large enough and a collection $\left\{Q_{\tau}^{k, \nu}:\right.$ $\left.k \in \mathbb{Z}_{+}, \tau \in I_{k}, \nu=1, \ldots, N(k, \tau)\right\}$ of open subsets satisfy the conditions as above.

(i) If $\max (d /(d+\theta), d /(d+\theta+s))<p \leq \infty, 0<q \leq \infty$ and $f \in$ $(\stackrel{\mathcal{G}}{ }(\beta, \gamma))^{\prime} \cap B_{p q}^{s}(X)$ with $\max \left(0, s_{+},-s+d(1 / p-1)_{+}\right)<\beta<\theta$ and $d(1 / p-1)_{+}$ $<\gamma<\theta$, then there exist a sequence $\lambda=\left\{\lambda_{Q_{\tau}^{k, \nu}}\right\}_{Q_{\tau}^{k, \nu} \in \mathbb{J}}$, $\varepsilon$-smooth blocks $a_{Q_{\tau}^{0, \nu}}$ for $\tau \in I_{0}$ and $\nu=1, \ldots, N(0, \tau)$, and $\varepsilon$-smooth atoms $a_{Q_{\tau}^{k, \nu}}$ for $k \in \mathbb{N}$, $\tau \in I_{k}$ and $\nu=1, \ldots, N(k, \tau), \varepsilon \in(|s|, \theta]$, such that

$$
f=\sum_{k=0}^{\infty} \sum_{\tau \in I_{k}} \sum_{\nu=1}^{N(k, \tau)} \lambda_{Q_{\tau}^{k, \nu}} a_{Q_{\tau}^{k, \nu}}
$$

with convergence both in the norm of $B_{p q}^{s}(X)$ and in $(\stackrel{\circ}{\mathcal{G}}(\beta, \gamma))^{\prime}$ when $\max (p, q)<\infty$, and only in $(\stackrel{\circ}{\mathcal{G}}(\beta, \gamma))^{\prime}$ when $\max (p, q)=\infty$, and

$$
\|\lambda\|_{b_{p q}^{s}(X)} \leq C\|f\|_{B_{p q}^{s}(X)} .
$$

(ii) If $\max (d /(d+\theta), d /(d+\theta+s))<p, q$ and $p<\infty, q \leq \infty$, and $f \in(\stackrel{\mathcal{G}}{(\beta, \gamma}))^{\prime} \cap F_{p q}^{s}(X)$ with $\max \left(0, s_{+},-s+d(1 / p-1)_{+}\right)<\beta<\theta$ and

$$
d(1 / p-1)_{+}<\gamma<\theta,
$$

then there exist a sequence $\lambda=\left\{\lambda_{Q_{\tau}^{k, \nu}}\right\}_{Q_{\tau}^{k, \nu} \in \mathbb{J}}$, $\varepsilon$-smooth blocks $a_{Q_{\tau}^{0, \nu}}$ for $\tau \in I_{0}$ and $\nu=1, \ldots, N(0, \tau)$, and $\varepsilon$-smooth atoms $a_{Q_{\tau}^{k, \nu}}$ for $k \in \mathbb{N}, \tau \in I_{k}$ and $\nu=1, \ldots, N(k, \tau), \varepsilon \in(|s|, \theta]$, such that (1.6) holds with convergence both in the norm of $F_{p q}^{s}(X)$ and in $(\stackrel{\mathcal{G}}{\mathcal{G}}(\beta, \gamma))^{\prime}$ when $q<\infty$, and only in $(\stackrel{\circ}{\mathcal{G}}(\beta, \gamma))^{\prime}$ when $q=\infty$, and

$$
\|\lambda\|_{f_{p q}^{s}(X)} \leq C\|f\|_{F_{p q}^{s}(X)} .
$$

The converse of Theorem 4 also holds. In fact, we have a general result. To state it, we recall the definition of smooth molecules and units in [9].

Definition 8. Fix $j \in \mathbb{N}$ and a collection of open subsets,

$$
\left\{Q_{\tau}^{k, \nu}: k \in \mathbb{Z}_{+}, \tau \in I_{k}, \nu=1, \ldots, N(k, \tau)\right\},
$$

satisfying the conditions as above. A function $u_{Q_{\tau}^{k, \nu}}$ defined on $X$ is said to be a $(\beta, \gamma)$-smooth molecule for a dyadic cube $Q_{\tau}^{k, \nu}$ if

(i) $\int_{X} u_{Q_{\tau}^{k, \nu}}(x) d \mu(x)=0$;

(ii) $\left|u_{Q_{\tau}^{k, \nu}}(x)\right| \leq \mu\left(Q_{\tau}^{k, \nu}\right)^{-1 / 2}\left(1+2^{k} \varrho\left(x, y_{\tau}^{k, \nu}\right)\right)^{-(d+\gamma)}$; 
(iii) $\left|u_{Q_{\tau}^{k, \nu}}(x)-u_{Q_{\tau}^{k, \nu}}\left(x^{\prime}\right)\right| \leq \mu\left(Q_{\tau}^{k, \nu}\right)^{-1 / 2-\beta / d} \varrho\left(x, x^{\prime}\right)^{\beta}$

$$
\times\left\{\frac{1}{\left(1+2^{k} \varrho\left(x, y_{\tau}^{k, \nu}\right)\right)^{d+\gamma}}+\frac{1}{\left(1+2^{k} \varrho\left(x^{\prime}, y_{\tau}^{k, \nu}\right)\right)^{d+\gamma}}\right\},
$$

where $y_{\tau}^{k, \nu}$ is the center of $Q_{\tau}^{k, \nu}$.

A function $u_{Q_{\tau}^{k, \nu}}$ defined on $X$ is said to be a $(\beta, \gamma)$-smooth unit for a dyadic cube $Q_{\tau}^{k, \nu}$ if $u_{Q_{\tau}^{k, \nu}}$ satisfies only (ii) and (iii) above.

Theorem 5. Let $s \in(-\theta, \theta), u_{Q_{\tau}^{0, \nu}}$ for $\tau \in I_{0}$ and $\nu=1, \ldots, N(0, \tau)$ be $a(\beta, \gamma)$-smooth unit, and $u_{Q_{\tau}^{k, \nu}}$ for $\tau \in I_{k}$ and $\nu=1, \ldots, N(k, \tau)$ be $a(\beta, \gamma)$ smooth molecule with $s_{+}<\beta<\theta, \max \left(d(1 / p-1)_{+},-s+d(1 / p-1)_{+}\right)<$ $\gamma<\theta$ for the spaces $b_{p q}^{s}(X)$, and

$$
\max \left(d(1 / \min (p, q)-1)_{+},-s+d(1 / \min (p, q)-1)_{+}\right)<\gamma<\theta
$$

for the spaces $f_{p q}^{s}(X)$. then

(i) If $\max (d /(d+\theta), d /(d+\theta+s))<p \leq \infty, 0<q \leq \infty$ and $\lambda \in b_{p q}^{s}(X)$,

$$
f=\sum_{k=0}^{\infty} \sum_{\tau \in I_{k}} \sum_{\nu=1}^{N(k, \tau)} \lambda_{Q_{\tau}^{k, \nu}} u_{Q_{\tau}^{k, \nu}}
$$

holds both in the norm of $B_{p q}^{s}(X)$ and in $\left(\stackrel{\mathcal{G}}{(}\left(\beta_{1}, \gamma_{1}\right)\right)^{\prime}$ with

$$
\max \left(0, s_{+},-s+d(1 / p-1)_{+}\right)<\beta_{1}<\theta
$$

and $0<\gamma_{1}<\theta$ when $\max (p, q)<\infty$, and only in $\left(\stackrel{\mathcal{G}}{\mathcal{G}}\left(\beta_{1}, \gamma_{1}\right)\right)^{\prime}$ when $\max (p, q)=\infty$, and

$$
\|f\|_{B_{p q}^{s}(X)} \leq C\|\lambda\|_{b_{p q}^{s}(X)} .
$$

(ii) If $\max (d /(d+\theta), d /(d+\theta+s))<p, q$ and $p<\infty, q \leq \infty$, and $\lambda \in f_{p q}^{s}(X)$, then (1.9) holds both in the norm of $F_{p q}^{s}(X)$ and in $\left(\stackrel{\circ}{\mathcal{G}}\left(\beta_{1}, \gamma_{1}\right)\right)^{\prime}$ with $\max \left(0, s_{+},-s+d(1 / p-1)_{+}\right)<\beta_{1}<\theta$ and $0<\gamma_{1}<\theta$ when $q<\infty$, and only in $\left(\mathcal{G}\left(\beta_{1}, \gamma_{1}\right)\right)^{\prime}$ when $q=\infty$, and

$$
\|f\|_{F_{p q}^{s}(X)} \leq C\|\lambda\|_{f_{p q}^{s}(X)} .
$$

2. Proofs. The basic tools we will use are the following inhomogeneous Calderón reproducing formulae ([11]) and the Plancherel-Pólya inequalities ([4]).

Lemma $2([11])$. Suppose that $\left\{E_{k}\right\}_{k=0}^{\infty}$ is as in Theorem 1. Then there exist functions $\widetilde{E}_{Q_{\tau}^{0, \nu}}, \widetilde{F}_{Q_{\tau}^{0, \nu}}, \tau \in I_{0}$ and $\nu=1, \ldots, N(0, \tau), \widetilde{E}_{k}(x, y)$ and $\widetilde{F}_{k}(x, y), k \in \mathbb{N}$, such that for any fixed $y_{\tau}^{k, \nu} \in Q_{\tau}^{k, \nu}, k \in \mathbb{N}, \tau \in I_{k}$ and $\nu \in$ 
$\{1, \ldots, N(k, \tau)\}$ and all $f \in\left(\stackrel{\circ}{\mathcal{G}}\left(\beta_{1}, \gamma_{1}\right)\right)^{\prime}$ with $0<\beta_{1}<\theta$ and $0<\gamma_{1}<\theta$,

$$
\begin{aligned}
f(x)= & \sum_{\tau \in I_{0}} \sum_{\nu=1}^{N(0, \tau)} \mu\left(Q_{\tau}^{0, \nu}\right) m_{Q_{\tau}^{0, \nu}}\left(E_{0}(f)\right) \widetilde{E}_{Q_{\tau}^{0, \nu}}(x) \\
& +\sum_{k=1}^{\infty} \sum_{\tau \in I_{k}} \sum_{\nu=1}^{N(k, \tau)} \mu\left(Q_{\tau}^{k, \nu}\right) E_{k}(f)\left(y_{\tau}^{k, \nu}\right) \widetilde{E}_{k}\left(x, y_{\tau}^{k, \nu}\right) \\
= & \sum_{\tau \in I_{0}} \sum_{\nu=1}^{N(0, \tau)} \mu\left(Q_{\tau}^{0, \nu}\right) \widetilde{F}_{Q_{\tau}^{0, \nu}}(f) m_{Q_{\tau}^{0, \nu}}\left(E_{0}(x, \cdot)\right) \\
& +\sum_{k=1}^{\infty} \sum_{\tau \in I_{k}} \sum_{\nu=1}^{N(k, \tau)} \mu\left(Q_{\tau}^{k, \nu}\right) \widetilde{F}_{k}(f)\left(y_{\tau}^{k, \nu}\right) E_{k}\left(x, y_{\tau}^{k, \nu}\right),
\end{aligned}
$$

where the series converge in $\left(\stackrel{\circ}{\mathcal{G}}\left(\beta_{1}^{\prime}, \gamma_{1}^{\prime}\right)\right)^{\prime}$ for $\beta_{1}<\beta_{1}^{\prime}<\theta$ and $\gamma_{1}<\gamma_{1}^{\prime}<\theta$; $\widetilde{E}_{k}(x, y), k \in \mathbb{N}$, satisfies conditions (i) and (ii) of Definition 3 with $\varepsilon \in$ $(0, \theta)$, and

$$
\int_{X} \widetilde{E}_{k}(x, y) d \mu(x)=\int_{X} \widetilde{E}_{k}(x, y) d \mu(y)=0 ;
$$

$\widetilde{F}_{k}(x, y), k \in \mathbb{N}$, satisfies conditions (i) and (iii) of Definition 3 with $\varepsilon \in$ $(0, \theta)$, and

$$
\int_{X} \widetilde{F}_{k}(x, y) d \mu(x)=\int_{X} \widetilde{F}_{k}(x, y) d \mu(y)=0 ;
$$

$\operatorname{diam}\left(Q_{\tau}^{0, \nu}\right) \sim 2^{-j}$ for $\tau \in I_{0}, \nu=1, \ldots, N(0, \tau)$, and some $j \in \mathbb{N} ; \widetilde{E}_{Q_{\tau}^{0, \nu}}(x)$ for $\tau \in I_{0}$ and $\nu=1, \ldots, N(0, \tau)$ satisfies

(i) $\int_{X} \widetilde{E}_{Q_{\tau}^{0, \nu}}(x) d \mu(x)=1$,

(ii) for any given $\varepsilon \in(0, \theta)$, there is a constant $C>0$ such that

$$
\left|\widetilde{E}_{Q_{\tau}^{0, \nu}}(x)\right| \leq C \frac{1}{(1+\varrho(x, y))^{d+\varepsilon}}
$$

for all $x \in X$ and $y \in Q_{\tau}^{0, \nu}$ and

(iii) $\left|\widetilde{E}_{Q_{\tau}^{0, \nu}}(x)-\widetilde{E}_{Q_{\tau}^{0, \nu}}(z)\right| \leq C\left(\frac{\varrho(x, z)}{1+\varrho(x, y)}\right)^{\varepsilon} \frac{1}{(1+\varrho(x, y))^{d+\varepsilon}}$

for all $x, z \in X$ and all $y \in Q_{\tau}^{0, \nu}$ satisfying $\varrho(x, z) \leq \frac{1}{2 A}(1+\varrho(x, y))$; and $\widetilde{F}_{Q_{\tau}^{0, \nu}}(x)$ satisfies the same conditions as $\widetilde{E}_{Q_{\tau}^{0, \nu}}(x)$, and

$$
\widetilde{F}_{Q_{\tau}^{0, \nu}}(f)=\int_{X} \widetilde{F}_{Q_{\tau}^{0, \nu}}(y) f(y) d \mu(y)
$$


Moreover, $j$ can be any fixed large positive integer and the constant $C$ in (ii) and (iii) is independent of $j$.

Lemma $3([4])$. Let $s \in(-\theta, \theta)$. Let $\left\{S_{k}\right\}_{k=0}^{\infty}$ and $\left\{P_{k}\right\}_{k=0}^{\infty}$ be two approximations to the identity of order $\theta$ as in Definition $3, E_{k}=S_{k}-S_{k-1}$ and $D_{k}=P_{k}-P_{k-1}$ for $k \in \mathbb{N}$, and $E_{0}=S_{0}$ and $D_{0}=P_{0}$. Then there is a constant $C_{5}>0$ such that for all $f \in(\stackrel{\circ}{\mathcal{G}}(\beta, \gamma))^{\prime}$ with $|s|<\beta<\theta$ and $0<\gamma<\theta$

$$
\begin{aligned}
& \left\{\sum_{\tau \in I_{0}} \sum_{\nu=1}^{N(0, \tau)} \mu\left(Q_{\tau}^{0, \nu}\right)\left[m_{Q_{\tau}^{0, \nu}}\left(\left|D_{0}(f)\right|\right)\right]^{p}\right\}^{1 / p} \\
& \quad+\left\{\sum_{k=1}^{\infty} 2^{k s q}\left(\sum_{\tau \in I_{k}} \sum_{\nu=1}^{N(k, \tau)} \mu\left(Q_{\tau}^{k, \nu}\right)\left[\sup _{z \in Q_{\tau}^{k, \nu}}\left|D_{k}(f)(z)\right|\right]^{p}\right)^{q / p}\right\}^{1 / q} \\
& \leq C_{5}\left\{\sum_{\tau \in \bar{I}_{0}} \sum_{\nu=1}^{\bar{N}(0, \tau)} \mu\left(\bar{Q}_{\tau}^{0, \nu}\right)\left[m_{\bar{Q}_{\tau}^{0, \nu}}\left(\left|E_{0}(f)\right|\right)\right]^{p}\right\}^{1 / p} \\
& \quad+C_{5}\left\{\sum_{k=1}^{\infty} 2^{k s q}\left(\sum_{\tau \in \bar{I}_{k}} \sum_{\nu=1}^{\bar{N}(k, \tau)} \mu\left(\bar{Q}_{\tau}^{k, \nu}\right)\left[\inf _{z \in \bar{Q}_{\tau}^{k, \nu}}\left|E_{k}(f)(z)\right|\right]^{p}\right)^{q / p}\right\}^{1 / q}
\end{aligned}
$$

when $\max (d /(d+\theta), d /(d+\theta+s))<p \leq \infty$ and $0<q \leq \infty$, and

$$
\begin{aligned}
& \left\{\sum_{\tau \in I_{0}} \sum_{\nu=1}^{N(0, \tau)} \mu\left(Q_{\tau}^{0, \nu}\right)\left[m_{Q_{\tau}^{0, \nu}}\left(\left|D_{0}(f)\right|\right)\right]^{p}\right\}^{1 / p} \\
& \quad+\left\|\left\{\sum_{k=1}^{\infty} \sum_{\tau \in I_{k}} \sum_{\nu=1}^{N(k, \tau)}\left[2^{k s} \sup _{z \in Q_{\tau}^{k, \nu}}\left|D_{k}(f)(z)\right| \chi_{Q_{\tau}^{k, \nu}}\right]^{q}\right\}^{1 / q}\right\| \|_{L^{p}(X)} \\
& \leq C_{5}\left\{\sum_{\tau \in \bar{I}_{0}} \sum_{\nu=1}^{\bar{N}(0, \tau)} \mu\left(\bar{Q}_{\tau}^{0, \nu}\right)\left[m_{\bar{Q}_{\tau}^{0, \nu}}\left(\left|E_{0}(f)\right|\right)\right]^{p}\right\}^{1 / p} \\
& \quad+C_{5}\left\|\left\{\sum_{k=1}^{\infty} \sum_{\tau \in \bar{I}_{k}} \sum_{\nu=1}^{\bar{N}(k, \tau)}\left[2^{k s} \inf _{z \in \bar{Q}_{\tau}^{k, \nu}}\left|E_{k}(f)(z)\right| \chi_{\bar{Q}_{\tau}^{k, \nu}}\right]^{q}\right\}^{1 / q}\right\|_{L^{p}(X)}
\end{aligned}
$$

when $\max (d /(d+\theta), d /(d+\theta+s))<p, q$ and $p<\infty, q \leq \infty$, where $\operatorname{diam}\left(Q_{\tau}^{0, \nu}\right) \sim 2^{-j}, \operatorname{diam}\left(\bar{Q}_{\tau}^{0, \nu}\right) \sim 2^{-\bar{j}}, j$ and $\bar{j}$ are two fixed large positive integers, the constant $C_{5}$ depends only on $\bar{j}$ when $p<1$, and it is independent of $j$ and $\bar{j}$ when $1 \leq p \leq \infty$ in (2.2) and $1 \leq p<\infty$ in (2.3). 
Proof of Theorem 1. The proof is based on the fact that for all $k \in \mathbb{N}$,

$$
\begin{aligned}
\sum_{\tau \in I_{k}} \sum_{\nu=1}^{N(k, \tau)} \mu\left(Q_{\tau}^{k, \nu}\right)\left[\inf _{z \in Q_{\tau}^{k, \nu}}\left|E_{k}(f)(z)\right|\right]^{p} & \leq\left\|E_{k}(f)\right\|_{L^{p}(X)}^{p} \\
& \leq \sum_{\tau \in I_{k}} \sum_{\nu=1}^{N(k, \tau)} \mu\left(Q_{\tau}^{k, \nu}\right)\left[\sup _{z \in Q_{\tau}^{k, \nu}}\left|E_{k}(f)(z)\right|\right]^{p}
\end{aligned}
$$

and for all $x \in X$,

$$
\begin{aligned}
\sum_{\tau \in I_{k}} \sum_{\nu=1}^{N(k, \tau)}\left[\inf _{z \in Q_{\tau}^{k, \nu}}\left|E_{k}(f)(z)\right|\right] \chi_{Q_{\tau}^{k, \nu}}(x) & \leq\left|E_{k}(f)(x)\right| \\
& \leq \sum_{\tau \in I_{k}} \sum_{\nu=1}^{N(k, \tau)}\left[\sup _{z \in Q_{\tau}^{k, \nu}}\left|E_{k}(f)(z)\right|\right] \chi_{Q_{\tau}^{k, \nu}}(x) .
\end{aligned}
$$

Applying Lemma 3 yields Theorem 1.

To prove Theorem 2, we need the following lemma which can be found in [6, pp. 147-148] for $\mathbb{R}^{n}$ and [12, pp. 93] for spaces of homogeneous type.

LEMMA 4. Let $0<r \leq 1, k, \eta \in \mathbb{Z}_{+}$with $\eta \leq k$, and for any dyadic cube $Q_{\tau}^{k, \nu}$

$$
\left|f_{Q_{\tau}^{k, \nu}}(x)\right| \leq\left(1+2^{\eta} \varrho\left(x, y_{\tau}^{k, \nu}\right)\right)^{-d-\gamma},
$$

where $y_{\tau}^{k, \nu}$ is any point in $Q_{\tau}^{k, \nu}$ and $\gamma>d(1 / r-1)$. Then

$$
\begin{aligned}
\sum_{\tau \in I_{k}} \sum_{\nu=1}^{N(k, \tau)}\left|\lambda_{Q_{\tau}^{k, \nu}}\right|\left|f_{Q_{\tau}^{k, \nu}}(x)\right| \leq & C 2^{(k-\eta) d / r} \\
& \times\left[M\left(\sum_{\tau \in I_{k}} \sum_{\nu=1}^{N(k, \tau)}\left|\lambda_{Q_{\tau}^{k, \nu}}\right|^{r} \chi_{Q_{\tau}^{k, \nu}}\right)(x)\right]^{1 / r},
\end{aligned}
$$

where $C$ is independent of $x, k$ and $\eta$, and $M$ is the Hardy-Littlewood maximal operator on $X$.

Proof of Theorem 2. We first show (1.3). Since $1 \leq p \leq \infty$, by the Hölder inequality we have

$$
\begin{aligned}
& \left\{\sum_{\tau \in I_{0}} \sum_{\nu=1}^{N(0, \tau)} \mu\left(Q_{\tau}^{0, \nu}\right)\left[m_{Q_{\tau}^{0, \nu}}\left(\left|E_{0}(f)\right|\right)\right]^{p}\right\}^{1 / p} \\
& \quad \leq\left\{\sum_{\tau \in I_{0}} \sum_{\nu=1}^{N(0, \tau)} \int_{Q_{\tau}^{0, \nu}}\left|E_{0}(f)(z)\right|^{p} d \mu(z)\right\}^{1 / p} \leq\left\|E_{0}(f)\right\|_{L^{p}(X)},
\end{aligned}
$$


which shows that the left-hand side is dominated by the right-hand side in (1.3).

To establish the converse, we only need to show that for $f \in(\stackrel{\circ}{\mathcal{G}}(\beta, \gamma))^{\prime}$ with $|s|<\beta<\theta$ and $0<\gamma<\theta$,

$$
\begin{aligned}
\left\|E_{0}(f)\right\|_{L^{p}(X)}= & \left\{\sum_{\tau \in I_{0}} \sum_{\nu=1}^{N(0, \tau)} \int_{Q_{\tau}^{0, \nu}}\left|E_{0}(f)(x)\right|^{p} d \mu(x)\right\}^{1 / p} \\
\leq & \left\{\sum_{\tau \in I_{0}} \sum_{\nu=1}^{N(0, \tau)} \mu\left(Q_{\tau}^{0, \nu}\right)\left[\sup _{z \in Q_{\tau}^{0, \nu}}\left|E_{0}(f)(z)\right|\right]^{p}\right\}^{1 / p} \\
\leq & C\left\{\sum_{\tau \in I_{0}} \sum_{\nu=1}^{N(0, \tau)} \mu\left(Q_{\tau}^{0, \nu}\right)\left[m_{\bar{Q}_{\tau}^{0, \nu}}\left(\left|E_{0}(f)\right|\right)\right]^{p}\right\}^{1 / p} \\
& +C\left\{\sum_{k=1}^{\infty}\left[2^{k s}\left\|E_{k}(f)\right\|_{L^{p}(X)}\right]^{q}\right\}^{1 / q} .
\end{aligned}
$$

To prove the last inequality in (2.5), by the first expression in (2.1) of Lemma 2 we have

$$
\begin{aligned}
& \sup _{z \in Q_{\tau}^{0, \nu}}\left|E_{0}(f)(z)\right| \\
\leq & \sum_{\tau^{\prime} \in I_{0}} \sum_{\nu^{\prime}=1}^{N\left(0, \tau^{\prime}\right)} \mu\left(Q_{\tau^{\prime}}^{0, \nu^{\prime}}\right) m_{Q_{\tau^{\prime}}^{0, \nu^{\prime}}}\left(\left|E_{0}(f)\right|\right) \sup _{z \in Q_{\tau}^{0, \nu}}\left|E_{0}\left(\widetilde{E}_{Q_{\tau^{\prime}}^{0, \nu^{\prime}}}\right)(z)\right| \\
& +\sum_{k^{\prime}=1}^{\infty} \sum_{\tau^{\prime} \in I_{k^{\prime}}} \sum_{\nu^{\prime}=1}^{N\left(k^{\prime}, \tau^{\prime}\right)} \mu\left(Q_{\tau^{\prime}}^{k^{\prime}, \nu^{\prime}}\right)\left|E_{k^{\prime}}(f)\left(y_{\tau^{\prime}}^{k^{\prime}, \nu^{\prime}}\right)\right| \sup _{z \in Q_{\tau}^{0, \nu}}\left|E_{0}\left(\widetilde{E}_{k^{\prime}}\right)\left(z, y_{\tau^{\prime}}^{k^{\prime}, \nu^{\prime}}\right)\right| \\
= & G_{1}+G_{2},
\end{aligned}
$$

where

$$
E_{0}\left(\widetilde{E}_{k^{\prime}}\right)\left(z, y_{\tau^{\prime}}^{k^{\prime}, \nu^{\prime}}\right)=\int_{X} E_{0}(z, x) \widetilde{E}_{k^{\prime}}\left(x, y_{\tau^{\prime}}^{k^{\prime}, \nu^{\prime}}\right) d \mu(x) .
$$

By a proof similar to those of (1.6) in [9] and (3.9) in [8], we can verify

$$
\sup _{z \in Q_{\tau}^{0, \nu}}\left|E_{0}\left(\widetilde{E}_{Q_{\tau^{\prime}}^{0, \nu^{\prime}}}\right)(z)\right| \leq C \frac{1}{\left(1+\varrho\left(y_{\tau}^{0, \nu}, y_{\tau^{\prime}}^{0, \nu^{\prime}}\right)\right)^{d+\varepsilon^{\prime}}},
$$

and for $k^{\prime} \in \mathbb{N}$,

$$
\sup _{z \in Q_{\tau}^{0, \nu}}\left|E_{0}\left(\widetilde{E}_{k^{\prime}}\right)\left(z, y_{\tau^{\prime}}^{k^{\prime}, \nu^{\prime}}\right)\right| \leq C 2^{-k^{\prime} \varepsilon^{\prime}} \frac{1}{\left(1+\varrho\left(y_{\tau}^{0, \nu}, y_{\tau^{\prime}}^{0, \nu^{\prime}}\right)\right)^{d+\varepsilon^{\prime}}},
$$

where $\varepsilon^{\prime}$ can be any positive number in $(0, \theta)$. In what follows, we will choose $\varepsilon^{\prime} \in(0, \theta)$ such that $|s|<\varepsilon^{\prime}$. 
From (2.7) and the Hölder inequality, it follows that

(2.9) $\left\{\sum_{\tau \in I_{0}} \sum_{\nu=1}^{N(0, \tau)} \mu\left(Q_{\tau}^{0, \nu}\right)\left[G_{1}\right]^{p}\right\}^{1 / p}$

$\leq C\left\{\sum_{\tau \in I_{0}} \sum_{\nu=1}^{N(0, \tau)} \mu\left(Q_{\tau}^{0, \nu}\right)\left(\sum_{\tau^{\prime} \in I_{0}} \sum_{\nu^{\prime}=1}^{N\left(0, \tau^{\prime}\right)} \mu\left(Q_{\tau^{\prime}}^{0, \nu^{\prime}}\right)\left[m_{Q_{\tau^{\prime}}^{0, \nu^{\prime}}}\left(\left|E_{0}(f)\right|\right)\right]^{p}\right.\right.$

$\left.\left.\times \frac{1}{\left(1+\varrho\left(y_{\tau}^{0, \nu}, y_{\tau^{\prime}}^{0, \nu^{\prime}}\right)\right)^{d+\varepsilon^{\prime}}}\right)\left[\int_{X} \frac{1}{\left(1+\varrho\left(y_{\tau}^{0, \nu}, y\right)\right)^{d+\varepsilon^{\prime}}} d \mu(y)\right]^{p / p^{\prime}}\right\}^{1 / p}$

$\leq C\left\{\sum_{\tau^{\prime} \in I_{0}} \sum_{\nu^{\prime}=1}^{N\left(0, \tau^{\prime}\right)} \mu\left(Q_{\tau^{\prime}}^{0, \nu^{\prime}}\right)\left[m_{Q_{\tau^{\prime}}^{0, \nu^{\prime}}}\left(\left|E_{0}(f)\right|\right)\right]^{p} \int_{X} \frac{1}{\left(1+\varrho\left(x, y_{\tau^{\prime}}^{0, \nu^{\prime}}\right)\right)^{d+\varepsilon^{\prime}}} d \mu(x)\right\}^{1 / p}$

$\leq C\left\{\sum_{\tau^{\prime} \in I_{0}} \sum_{\nu^{\prime}=1}^{N\left(0, \tau^{\prime}\right)} \mu\left(Q_{\tau^{\prime}}^{0, \nu^{\prime}}\right)\left[m_{Q_{\tau^{\prime}}^{0, \nu^{\prime}}}\left(\left|E_{0}(f)\right|\right)\right]^{p}\right\}^{1 / p} ;$

while from (2.8) and the Hölder inequality, it follows that

$$
\begin{aligned}
& \left\{\sum_{\tau \in I_{0}} \sum_{\nu=1}^{N(0, \tau)} \mu\left(Q_{\tau}^{0, \nu}\right)\left[G_{2}\right]^{p}\right\}^{1 / p} \\
& \leq C\left\{\sum _ { \tau \in I _ { 0 } } \sum _ { \nu = 1 } ^ { N ( 0 , \tau ) } \mu ( Q _ { \tau } ^ { 0 , \nu } ) \left[\sum_{k^{\prime}=1}^{\infty} 2^{-k^{\prime} \varepsilon_{1} p} \sum_{\tau^{\prime} \in I_{k^{\prime}}} \sum_{\nu^{\prime}=1}^{N\left(k^{\prime}, \tau^{\prime}\right)} \mu\left(Q_{\tau^{\prime}}^{k^{\prime}, \nu^{\prime}}\right)\right.\right. \\
& \left.\times\left|E_{k^{\prime}}(f)\left(y_{\tau^{\prime}}^{k^{\prime}, \nu^{\prime}}\right)\right|^{p} \frac{1}{\left(1+\varrho\left(y_{\tau}^{0, \nu}, y_{\tau^{\prime}}^{k^{\prime}, \nu^{\prime}}\right)\right)^{d+\varepsilon^{\prime}}}\right] \\
& \left.\times\left[\sum_{k^{\prime}=1}^{\infty} 2^{-k^{\prime} \varepsilon_{1} p} \int_{X} \frac{1}{\left(1+\varrho\left(y_{\tau}^{0, \nu}, y\right)\right)^{d+\varepsilon^{\prime}}} d \mu(y)\right]^{p / p^{\prime}}\right\}^{1 / p} \\
& \leq C\left\{\sum_{k^{\prime}=1}^{\infty} 2^{-k^{\prime}\left(\varepsilon_{1}+s\right) p} 2^{k^{\prime} s p} \sum_{\tau^{\prime} \in I_{k^{\prime}}} \sum_{\nu^{\prime}=1}^{N\left(k^{\prime}, \tau^{\prime}\right)} \mu\left(Q_{\tau^{\prime}}^{k^{\prime}, \nu^{\prime}}\right)\left|E_{k^{\prime}}(f)\left(y_{\tau^{\prime}}^{k^{\prime}, \nu^{\prime}}\right)\right|^{p}\right. \\
& \left.\times \int_{X} \frac{1}{\left(1+\varrho\left(z, y_{\tau^{\prime}}^{k^{\prime}, \nu^{\prime}}\right)\right)^{d+\varepsilon^{\prime}}} d \mu(z)\right\}^{1 / p} \\
& \leq C\left\{\sum_{k^{\prime}=1}^{\infty} 2^{k^{\prime} s p}\left[\sum_{\tau^{\prime} \in I_{k^{\prime}}} \sum_{\nu^{\prime}=1}^{N\left(k^{\prime}, \tau^{\prime}\right)} \mu\left(Q_{\tau^{\prime}}^{k^{\prime}, \nu^{\prime}}\right)\left|E_{k^{\prime}}(f)\left(y_{\tau^{\prime}}^{k^{\prime}, \nu^{\prime}}\right)\right|^{p}\right]^{q / p}\right\}^{1 / q} \\
& \leq C\left\{\sum_{k^{\prime}=1}^{\infty}\left[2^{k^{\prime} s}\left\|E_{k^{\prime}}(f)\right\|_{L^{p}(X)}\right]^{q}\right\}^{1 / q},
\end{aligned}
$$


where we chose $\varepsilon_{1}, \varepsilon_{2}>0$ such that $\varepsilon^{\prime}=\varepsilon_{1}+\varepsilon_{2}$ and $\varepsilon_{1}>|s|$, and we used the arbitrariness of $y_{\tau^{\prime}}^{k^{\prime}, \nu^{\prime}}$ in the last step, while in the second-to-last step, we used the following well-known inequality: for $t \in(0,1]$ and $a_{j} \in \mathbb{C}$,

$$
\left(\sum_{j}\left|a_{j}\right|\right)^{t} \leq \sum_{j}\left|a_{j}\right|^{t} .
$$

Now (2.9) and (2.10) yield (2.5). This shows (1.3).

To verify (1.4), using the estimate of (2.4), we can first easily see that the left-hand side of (1.4) is dominated by its right-hand side. To establish the converse, by $(2.5)$, we only need to show that for $f \in(\stackrel{\circ}{\mathcal{G}}(\beta, \gamma))^{\prime}$ with $|s|<\beta<\theta$ and $0<\gamma<\theta$,

$$
\begin{aligned}
&\left\{\sum_{\tau \in I_{0}} \sum_{\nu=1}^{N(0, \tau)} \mu\left(Q_{\tau}^{0, \nu}\right)\left[\sup _{z \in Q_{\tau}^{0, \nu}}\left|E_{0}(f)(z)\right|\right]^{p}\right\}^{1 / p} \\
& \leq C\left\{\sum_{\tau \in I_{0}} \sum_{\nu=1}^{N(0, \tau)} \mu\left(Q_{\tau}^{0, \nu}\right)\left[m_{Q_{\tau}^{0, \nu}}\left(\left|E_{0}(f)\right|\right)\right]^{p}\right\}^{1 / p} \\
&+C\left\|\left\{\sum_{k=1}^{\infty}\left[2^{k s}\left|E_{k}(f)\right|\right]^{q}\right\}^{1 / q}\right\|_{L^{p}(X)} \cdot
\end{aligned}
$$

To prove (2.12), we control $\sup _{z \in Q_{\tau}^{0, \nu}}\left|E_{0}(f)(z)\right|$ by $G_{1}+G_{2}$ as in (2.6). By Lemma 4, (2.8) and the Fefferman-Stein vector-valued maximal function inequality in [5], we estimate the term related to $G_{2}$ by

$$
\begin{aligned}
& \left\{\sum_{\tau \in I_{0}} \sum_{\nu=1}^{N(0, \tau)} \mu\left(Q_{\tau}^{0, \nu}\right)\left[G_{2}\right]^{p}\right\}^{1 / p} \\
& \leq C\left\{\int _ { X } \sum _ { \tau \in I _ { 0 } } \sum _ { \nu = 1 } ^ { N ( 0 , \tau ) } \chi _ { Q _ { \tau } ^ { 0 , \nu } } ( x ) \left[\sum_{k^{\prime}=1}^{\infty} \sum_{\tau^{\prime} \in I_{k^{\prime}}} \sum_{\nu^{\prime}=1}^{N\left(k^{\prime}, \tau^{\prime}\right)} 2^{-k^{\prime}\left(\varepsilon^{\prime}+d\right)}\left|E_{k^{\prime}}(f)\left(y_{\tau^{\prime}}^{k^{\prime}, \nu^{\prime}}\right)\right|\right.\right. \\
& \left.\left.\times \frac{1}{\left(1+\varrho\left(x, y_{\tau^{\prime}}^{k^{\prime}, \nu^{\prime}}\right)\right) d+\varepsilon^{\prime}}\right]^{p} d \mu(x)\right\}^{1 / p} \\
& \leq C \| \sum_{k^{\prime}=1}^{\infty} 2^{-k^{\prime}\left(\varepsilon^{\prime}+s+d-d / r\right)} \\
& \times\left(M\left[\sum_{\tau^{\prime} \in I_{k^{\prime}}} \sum_{\nu^{\prime}=1}^{N\left(k^{\prime}, \tau^{\prime}\right)} 2^{k^{\prime} s r}\left|E_{k^{\prime}}(f)\left(y_{\tau^{\prime}}^{k^{\prime}, \nu^{\prime}}\right)\right|^{r} \chi_{Q_{\tau^{\prime}}^{k^{\prime}, \nu^{\prime}}}\right]\right)^{1 / r} \|_{L^{p}(X)} \\
& \leq C\left\|\left\{\sum_{k^{\prime}=1}^{\infty}\left(M\left[\sum_{\tau^{\prime} \in I_{k^{\prime}}} \sum_{\nu^{\prime}=1}^{N\left(k^{\prime}, \tau^{\prime}\right)} 2^{k^{\prime} s r}\left|E_{k^{\prime}}(f)\left(y_{\tau^{\prime}}^{k^{\prime}, \nu^{\prime}}\right)\right|^{r} \chi_{Q_{\tau^{\prime}}^{k^{\prime}, \nu^{\prime}}}\right]\right)^{q / r}\right\}^{1 / q}\right\|_{L^{p}(X)}
\end{aligned}
$$




$$
\begin{aligned}
& \leq C\left\|\left\{\sum_{k^{\prime}=1}^{\infty} \sum_{\tau^{\prime} \in I_{k^{\prime}}} \sum_{\nu^{\prime}=1}^{N\left(k^{\prime}, \tau^{\prime}\right)}\left[2^{k^{\prime} s}\left|E_{k^{\prime}}(f)\left(y_{\tau^{\prime}}^{k^{\prime}, \nu^{\prime}}\right)\right| \chi_{Q_{\tau^{\prime}}^{k^{\prime}, \nu^{\prime}}}\right]^{q}\right\}^{1 / q}\right\|_{L^{p}(X)} \\
& \leq C\left\|\left\{\sum_{k^{\prime}=1}^{\infty}\left[2^{k^{\prime} s}\left|E_{k^{\prime}}(f)\left(y_{\tau^{\prime}}^{k^{\prime}, \nu^{\prime}}\right)\right|\right]^{q}\right\}^{1 / q}\right\|_{L^{p}(X)},
\end{aligned}
$$

where we chose $r \in(0,1)$ such that $r>d /\left(d+s+\varepsilon^{\prime}\right)$, and in the last step we used the arbitrariness of $y_{\tau^{\prime}}^{k^{\prime}, \nu^{\prime}}$. Now, (2.10) and (2.13) prove (2.12). This shows (1.4).

From the smooth molecule and unit characterization of the spaces $F_{p q}^{s}(X)$ when $d /(d+\theta)<p<\infty$ and $1 \leq q<\infty$ (see Theorems 2.1 and 2.2 in [10]), and Theorems 4 and 5 below, it is easy to see that (1.5) holds.

This finishes the proof of Theorem 2.

From the proof of Theorem 1 in [4] (see also the proofs of (2.5) and (2.12) above), we can easily deduce the following lemma which is Remark 1 in [4].

LEMMA 5. With the notation as in Lemma 2,

$$
\begin{aligned}
& \left\{\sum_{\tau \in I_{0}} \sum_{\nu=1}^{N(0, \tau)} \mu\left(Q_{\tau}^{0, \nu}\right)\left|\widetilde{F}_{Q_{\tau}^{0, \nu}}(f)\right|^{p}\right\}^{1 / p} \\
& \quad+\left\{\sum_{k=1}^{\infty} 2^{k s q}\left(\sum_{\tau \in I_{k}} \sum_{\nu=1}^{N(k, \tau)} \mu\left(Q_{\tau}^{k, \nu}\right)\left[\sup _{z \in Q_{\tau}^{k, \nu}}\left|\widetilde{F}_{k}(f)(z)\right|\right]^{p}\right)^{q / p}\right\}^{1 / q} \leq C\|f\|_{B_{p q}^{s}(X)}
\end{aligned}
$$

when $\max (d /(d+\theta), d /(d+\theta+s))<p \leq \infty$ and $0<q \leq \infty$, and

$$
\begin{aligned}
& \left\{\sum_{\tau \in I_{0}} \sum_{\nu=1}^{N(0, \tau)} \mu\left(Q_{\tau}^{0, \nu}\right)\left|\widetilde{F}_{Q_{\tau}^{0, \nu}}(f)\right|^{p}\right\}^{1 / p} \\
& \quad+\left\|\left\{\sum_{k=1}^{\infty} \sum_{\tau \in I_{k}} \sum_{\nu=1}^{N(k, \tau)}\left[2^{k s} \sup _{z \in Q_{\tau}^{k, \nu}}\left|\widetilde{F}_{k}(f)(z)\right| \chi_{Q_{\tau}^{k, \nu}}\right]^{q}\right\}^{1 / q}\right\|_{L^{p}(X)} \leq C\|f\|_{F_{p q}^{s}(X)}
\end{aligned}
$$

when $\max (d /(d+\theta), d /(d+\theta+s))<p, q$ and $p<\infty, q \leq \infty$.

Proof of Theorem 3. Suppose $f \in\left(\stackrel{\circ}{\mathcal{G}}\left(\beta_{1}, \gamma_{1}\right)\right)^{\prime}$ and $h \in \mathcal{G}(\theta, \theta)$. By Lemma 2, we have

$$
\begin{aligned}
\langle f, h\rangle= & \sum_{\tau \in I_{0}} \sum_{\nu=1}^{N(0, \tau)} \mu\left(Q_{\tau}^{0, \nu}\right) \widetilde{F}_{Q_{\tau}^{0, \nu}}(f) \int_{X} m_{Q_{\tau}^{0, \nu}}\left(E_{0}(x, \cdot)\right) h(x) d \mu(x) \\
& +\sum_{k=1}^{\infty} \sum_{\tau \in I_{k}} \sum_{\nu=1}^{N(k, \tau)} \mu\left(Q_{\tau}^{k, \nu}\right) \widetilde{F}_{k}(f)\left(y_{\tau}^{k, \nu}\right)\left\langle E_{k}\left(\cdot, y_{\tau}^{k, \nu}\right), h\right\rangle \\
= & K_{1}+K_{2} .
\end{aligned}
$$


We first verify that for $\tau \in I_{0}$ and $\nu=1, \ldots, N(0, \tau)$,

$$
\begin{aligned}
&\left|\int_{X} m_{Q_{\tau}^{0, \nu}}\left(E_{0}(x, \cdot)\right) h(x) d \mu(x)\right| \\
& \leq C\|h\|_{\mathcal{G}\left(\beta_{2}, \gamma_{2}\right)} \inf _{y \in Q_{\tau}^{0, \nu}} \frac{1}{\left(1+\varrho\left(y, x_{0}\right)\right)^{d+\gamma_{2}}},
\end{aligned}
$$

and for $k \in \mathbb{N}, \tau \in I_{k}$ and $\nu=1, \ldots, N(k, \tau)$,

$$
\left|\left\langle E_{k}\left(\cdot, y_{\tau}^{k, \nu}\right), h\right\rangle\right| \leq C 2^{-k \beta_{2}}\|h\|_{\mathcal{G}\left(\beta_{2}, \gamma_{2}\right)} \inf _{y \in Q_{\tau}^{k, \nu}} \frac{1}{\left(1+\varrho\left(y, x_{0}\right)\right)^{d+\gamma_{2}}},
$$

where $C$ is independent of $n$.

To prove (2.15), we have

$$
\begin{aligned}
& \left|\int_{X} m_{Q_{\tau}^{0, \nu}}\left(E_{0}(x, \cdot)\right) h(x) d \mu(x)\right| \\
& \leq \sup _{y \in Q_{\tau}^{0, \nu}}\left|\int_{X} E_{0}(x, y) h(x) d \mu(x)\right| \\
& \leq C\|h\|_{\mathcal{G}\left(\beta_{2}, \gamma_{2}\right)} \sup _{y \in Q_{\tau}^{0, \nu}} \int_{\left\{x: \varrho(x, y) \leq C_{1}\right\}} \frac{1}{\left(1+\varrho\left(x, x_{0}\right)\right)^{d+\gamma_{2}}} d \mu(x) \\
& \leq C\|h\|_{\mathcal{G}\left(\beta_{2}, \gamma_{2}\right)} \\
& \quad \times \sup _{y \in Q_{\tau}^{0, \nu}}\left[\chi_{\left\{y: \varrho\left(y, x_{0}\right) \leq 2 A C_{1}\right\}}(y)+\chi_{\left\{y: \varrho\left(y, x_{0}\right)>2 A C_{1}\right\}}(y) \frac{1}{\left(1+\varrho\left(y, x_{0}\right)\right)^{d+\gamma_{2}}}\right] \\
& \leq C\|h\|_{\mathcal{G}\left(\beta_{2}, \gamma_{2}\right)} \sup _{y \in Q_{\tau}^{0, \nu}} \frac{1}{\left(1+\varrho\left(y, x_{0}\right)\right)^{d+\gamma_{2}}} \\
& \leq C\|h\|_{\mathcal{G}\left(\beta_{2}, \gamma_{2}\right)} \inf _{y \in Q_{\tau}^{0, \nu}} \frac{1}{\left(1+\varrho\left(y, x_{0}\right)\right)^{d+\gamma_{2}}} .
\end{aligned}
$$

That is, (2.15) holds.

For (2.16), we have

$$
\begin{aligned}
& \left|\left\langle E_{k}\left(\cdot, y_{\tau}^{k, \nu}\right), h\right\rangle\right|=\left|\int_{X} E_{k}\left(x, y_{\tau}^{k, \nu}\right)[h(x)-h(y)] d \mu(x)\right| \\
& \quad \leq C 2^{-k \beta_{2}}\|h\|_{\mathcal{G}\left(\beta_{2}, \gamma_{2}\right)} \int_{\left\{x: \varrho\left(x, y_{\tau}^{k, \nu}\right) \leq 3 C_{1} 2^{-k}\right\}} \frac{\varrho\left(x, y_{\tau}^{k, \nu}\right)^{\beta_{2}}}{\left(1+\varrho\left(y_{\tau}^{k, \nu}, x_{0}\right)\right)^{d+\gamma_{2}+\beta_{2}}} d \mu(x) \\
& \quad \leq C 2^{-k \beta_{2}}\|h\|_{\mathcal{G}\left(\beta_{2}, \gamma_{2}\right)} \frac{1}{\left(1+\varrho\left(y_{\tau}^{k, \nu}, x_{0}\right)\right)^{d+\gamma_{2}+\beta_{2}}} \\
& \quad \leq C 2^{-k \beta_{2}}\|h\|_{\mathcal{G}\left(\beta_{2}, \gamma_{2}\right)} \inf _{y \in Q_{\tau}^{k, \nu}} \frac{1}{\left(1+\varrho\left(y, x_{0}\right)\right)^{d+\gamma_{2}}} .
\end{aligned}
$$

Thus, (2.16) also holds. 
Let us now prove (i) of Theorem 3. By Lemma 5, the Hölder inequality, (2.11), (2.15) and (2.16), we obtain

$$
\begin{aligned}
& \left|K_{1}\right| \leq C\|h\|_{\mathcal{G}\left(\beta_{2}, \gamma_{2}\right)}\left\{\begin{array}{c}
{\left[\sum_{\tau \in I_{0}} \sum_{\nu=1}^{N(0, \tau)} \mu\left(Q_{\tau}^{0, \nu}\right)\left|\widetilde{F}_{Q_{\tau}^{0, \nu}}(f)\right|^{p}\right]^{1 / p}, \quad p<1,} \\
{\left[\sum_{\tau \in I_{0}} \sum_{\nu=1}^{N(0, \tau)} \mu\left(Q_{\tau}^{0, \nu}\right)\left|\widetilde{F}_{Q_{\tau}^{0, \nu}}(f)\right|^{p}\right]^{1 / p}} \\
\quad \times\left[\int_{X} \frac{1}{\left(1+\varrho\left(y, x_{0}\right)\right)^{\left(d+\gamma_{2}\right) p^{\prime}}} d \mu(y)\right]^{1 / p^{\prime}}, \\
1 \leq p \leq \infty,
\end{array}\right. \\
& \leq C\|f\|_{B_{p q}^{s}(X)}\|h\|_{\mathcal{G}\left(\beta_{2}, \gamma_{2}\right)} \text {; }
\end{aligned}
$$

and

$\left|K_{2}\right| \leq C\|h\|_{\mathcal{G}\left(\beta_{2}, \gamma_{2}\right)}\left\{\begin{array}{l}\sum_{k=1}^{\infty} 2^{-k \beta_{2}}\left[\sum_{\tau \in I_{k}} \sum_{\nu=1}^{N(k, \tau)} \mu\left(Q_{\tau}^{k, \nu}\right)^{p}\left|\widetilde{F}_{k}(f)\left(y_{\tau}^{k, \nu}\right)\right|^{p}\right]^{1 / p}, p<1, \\ \sum_{k=1}^{\infty} 2^{-k \beta_{2}}\left[\sum_{\tau \in I_{k}} \sum_{\nu=1}^{N(k, \tau)} \mu\left(Q_{\tau}^{k, \nu}\right)\left|\widetilde{F}_{k}(f)\left(y_{\tau}^{k, \nu}\right)\right|^{p}\right]^{1 / p} \\ \quad \times\left[\int_{X} \frac{1}{\left(1+\varrho\left(y, x_{0}\right)\right)^{\left(d+\gamma_{2}\right) p^{\prime}}} d \mu(y)\right]^{1 / p^{\prime}}, \quad 1 \leq p \leq \infty,\end{array}\right.$

$$
\leq C\|h\|_{\mathcal{G}\left(\beta_{2}, \gamma_{2}\right)}\left\{\begin{array}{l}
\sum_{k=1}^{\infty} 2^{-k\left[\beta_{2}+s-d(1 / p-1)\right]} 2^{k s} \\
\quad \times\left[\sum_{\tau \in I_{k}} \sum_{\nu=1}^{N(k, \tau)} \mu\left(Q_{\tau}^{k, \nu}\right)\left|\widetilde{F}_{k}(f)\left(y_{\tau}^{k, \nu}\right)\right|^{p}\right]^{1 / p}, p<1, \\
\sum_{k=1}^{\infty} 2^{-k\left(\beta_{2}+s\right)} 2^{k s} \\
\quad \times\left[\sum_{\tau \in I_{k}} \sum_{\nu=1}^{N(k, \tau)} \mu\left(Q_{\tau}^{k, \nu}\right)\left|\widetilde{F}_{k}(f)\left(y_{\tau}^{k, \nu}\right)\right|^{p}\right]^{1 / p}, 1 \leq p \leq \infty
\end{array}\right.
$$

$\leq C\|f\|_{B_{p q}^{s}(X)}\|h\|_{\mathcal{G}\left(\beta_{2}, \gamma_{2}\right)}$,

where we used $\beta_{2}>\max \left(0, s_{+},-s+d(1 / p-1)_{+}\right)$and the arbitrariness of $y_{\tau}^{k, \nu}$.

From (2.14)-(2.16), it follows that for all $h \in \mathcal{G}(\theta, \theta)$,

$$
|\langle f, h\rangle| \leq C\|f\|_{B_{p q}^{s}(X)}\|h\|_{\mathcal{G}\left(\beta_{2}, \gamma_{2}\right)} .
$$


Suppose now $h \in \stackrel{\circ}{\mathcal{G}}\left(\beta_{2}, \gamma_{2}\right)$. We choose $h_{n} \in \mathcal{G}(\theta, \theta)$ for all $n \in \mathbb{N}$ such that

$$
\left\|h_{n}-h\right\|_{\mathcal{G}\left(\beta_{2}, \gamma_{2}\right)} \rightarrow 0
$$

as $n \rightarrow \infty$. The estimate of (2.19) shows that for all $n, m \in \mathbb{N}$,

$$
\left|\left\langle f, h_{n}-h_{m}\right\rangle\right| \leq C\|f\|_{B_{p q}^{s}(X)}\left\|h_{n}-h_{m}\right\|_{\mathcal{G}\left(\beta_{2}, \gamma_{2}\right)},
$$

which shows $\lim _{n \rightarrow \infty}\left\langle f, h_{n}\right\rangle$ exists and the limit is independent of the choice of $h_{n}$. Therefore, we define

$$
\langle f, h\rangle=\lim _{n \rightarrow \infty}\left\langle f, h_{n}\right\rangle .
$$

By (2.19), for all $h \in \stackrel{\circ}{\mathcal{G}}\left(\beta_{2}, \gamma_{2}\right)$,

$$
|\langle f, h\rangle| \leq C\|f\|_{B_{p q}^{s}(X)}\|h\|_{\mathcal{G}\left(\beta_{2}, \gamma_{2}\right)} .
$$

Thus, $f \in\left(\stackrel{\circ}{\mathcal{G}}\left(\beta_{2}, \gamma_{2}\right)\right)^{\prime}$. This finishes the proof of (i) of Theorem 3 .

The proof of (ii) can be obtained by using the fact that

$$
\|f\|_{B_{p, \max (p, q)}^{s}(X)} \leq C\|f\|_{F_{p q}^{s}(X)}
$$

see Proposition 2.3.2/2 in [15]. This finishes the proof of Theorem 3.

The key to the proof of Theorem 4 is also the discrete Calderón reproducing formulae.

Proof of Theorem 4. Let $f \in B_{p q}^{s}(X) \cap(\stackrel{\circ}{\mathcal{G}}(\beta, \gamma))^{\prime}$ with $s, p, q, \beta$ and $\gamma$ as in Theorem 4. Let $\left\{S_{k}\right\}_{k=0}^{\infty}$ be an approximation to the identity of order $\theta$ with compact support as in Remark 1 . Let $E_{k}=S_{k}-S_{k-1}$ for $k \in \mathbb{N}$ and $E_{0}=S_{0}$. For this $\left\{E_{k}\right\}_{k=0}^{\infty}$, by Lemma 2 , we have

$$
\begin{aligned}
f(x)= & \sum_{\tau \in I_{0}} \sum_{\nu=1}^{N(0, \tau)} \mu\left(Q_{\tau}^{0, \nu}\right) \widetilde{F}_{Q_{\tau}^{0, \nu}}(f) m_{Q_{\tau}^{0, \nu}}\left(E_{0}(x, \cdot)\right) \\
& +\sum_{k=1}^{\infty} \sum_{\tau \in I_{k}} \sum_{\nu=1}^{N(k, \tau)} \mu\left(Q_{\tau}^{k, \nu}\right) \widetilde{F}_{k}(f)\left(y_{\tau}^{k, \nu}\right) E_{k}\left(x, y_{\tau}^{k, \nu}\right),
\end{aligned}
$$

where all the other notation is as in Lemma 2, $y_{\tau}^{k, \nu}$ is the center of $Q_{\tau}^{k, \nu}$, and $(2.20)$ holds in $\left(\mathcal{G}\left(\beta^{\prime}, \gamma^{\prime}\right)\right)^{\prime}$ with $\beta<\beta^{\prime}<\theta$ and $\gamma<\gamma^{\prime}<\theta$. Let $\lambda_{Q_{\tau}^{0, \nu}}=\mu\left(Q_{\tau}^{0, \nu}\right)^{1 / 2} \widetilde{F}_{Q_{\tau}^{0, \nu}}(f)$ and

$$
a_{Q_{\tau}^{0, \nu}}(x)=\mu\left(Q_{\tau}^{0, \nu}\right)^{1 / 2} m_{Q_{\tau}^{0, \nu}}\left(E_{0}(x, \cdot)\right)
$$

for $\tau \in I_{0}$ and $\nu=1, \ldots, N(0, \tau)$, while $\lambda_{Q_{\tau}^{k, \nu}}=\mu\left(Q_{\tau}^{k, \nu}\right)^{1 / 2} \widetilde{F}_{k}(f)\left(y_{\tau}^{k, \nu}\right)$ and

$$
a_{Q_{\tau}^{k, \nu}}(x)=\mu\left(Q_{\tau}^{k, \nu}\right)^{1 / 2} E_{k}\left(x, y_{\tau}^{k, \nu}\right)
$$

for $k \in \mathbb{N}, \tau \in I_{k}$ and $\nu=1, \ldots, N(k, \tau)$. Then it is easy to check that $a_{Q_{\tau}^{0, \nu}}(x)$ for $\tau \in I_{0}$ and $\nu=1, \ldots, N(0, \tau)$ is an $\varepsilon$-smooth block for $Q_{\tau}^{0, \nu}$, 
and $a_{Q_{\tau}^{k, \nu}}(x)$ for $k \in \mathbb{N}, \tau \in I_{k}$ and $N=1, \ldots, N(k, \tau)$ is an $\varepsilon$-smooth atom for $Q_{\tau}^{k, \nu}$, multiplied with a normalizing constant. Moreover, by Lemma 5, we have

$$
\begin{aligned}
\|\lambda\|_{b_{p q}^{s}(X) \leq} & C\left\{\sum_{\tau \in I_{0}} \sum_{\nu=1}^{N(0, \tau)} \mu\left(Q_{\tau}^{0, \nu}\right)\left|\widetilde{F}_{Q_{\tau}^{0, \nu}}(f)\right|^{p}\right\}^{1 / p} \\
& +C\left\{\sum_{k=1}^{\infty} 2^{k s q}\left[\sum_{\tau \in I_{k}} \sum_{\nu=1}^{N(k, \tau)} \mu\left(Q_{\tau}^{k, \nu}\right)\left|\widetilde{F}_{k}(f)\left(y_{\tau}^{k, \nu}\right)\right|^{p}\right]^{q / p}\right\}^{1 / q} \\
\leq & C\left\{\sum_{\tau \in I_{0}} \sum_{\nu=1}^{N(0, \tau)} \mu\left(Q_{\tau}^{0, \nu}\right)\left[m_{Q_{\tau}^{0, \nu}}\left(\left|E_{0}(f)\right|\right)\right]^{p}\right\}^{1 / p} \\
& +C\left\{\sum_{k=1}^{\infty} 2^{k s q}\left(\sum_{\tau \in I_{k}} \sum_{\nu=1}^{N(k, \tau)} \mu\left(Q_{\tau}^{k, \nu}\right)\left[\inf _{z \in Q_{\tau}^{k, \nu}}\left|E_{k}(f)(z)\right|\right]^{p}\right)^{q / p}\right\}^{1 / q} \\
\leq & C\|f\|_{B_{p q}^{s}(X) .}
\end{aligned}
$$

That is, (1.7) holds. By (1.7) and the same argument as in Theorem 3, we can show that $(2.20)$ also holds in $(\mathcal{G}(\beta, \gamma))^{\prime}$. To see that the series in $(2.20)$ converge in the norm of $B_{p q}^{s}(X)$ if $\max (p, q)<\infty$, without loss of generality, we may suppose $I_{k}=\mathbb{N}$ for all $k \in \mathbb{Z}_{+}$. For $L \in \mathbb{N}$, we then define

$$
\begin{aligned}
f_{L}(x)= & \sum_{\tau=1}^{L} \sum_{\nu=1}^{N(0, \tau)} \mu\left(Q_{\tau}^{0, \nu}\right) \widetilde{F}_{Q_{\tau}^{0, \nu}}(f) m_{Q_{\tau}^{0, \nu}}\left(E_{0}(x, \cdot)\right) \\
& +\sum_{k=1}^{L} \sum_{\tau=1}^{L} \sum_{\nu=1}^{N(k, \tau)} \mu\left(Q_{\tau}^{k, \nu}\right) \widetilde{F}_{k}(f)\left(y_{\tau}^{k, \nu}\right) E_{k}\left(x, y_{\tau}^{k, \nu}\right) .
\end{aligned}
$$

Then it is easy to see that for all $L \in \mathbb{N}$,

$$
\begin{aligned}
f(x)-f_{L}(x)= & \sum_{\tau=L+1}^{\infty} \sum_{\nu=1}^{N(0, \tau)} \mu\left(Q_{\tau}^{0, \nu}\right) \widetilde{F}_{Q_{\tau}^{0, \nu}}(f) m_{Q_{\tau}^{0, \nu}}\left(E_{0}(x, \cdot)\right) \\
& +\sum_{k=L+1}^{\infty} \sum_{\tau \in \mathbb{N}} \sum_{\nu=1}^{N(k, \tau)} \mu\left(Q_{\tau}^{k, \nu}\right) \widetilde{F}_{k}(f)\left(y_{\tau}^{k, \nu}\right) E_{k}\left(x, y_{\tau}^{k, \nu}\right) \\
& +\sum_{k=1}^{L} \sum_{\tau=L+1}^{\infty} \sum_{\nu=1}^{N(k, \tau)} \mu\left(Q_{\tau}^{k, \nu}\right) \widetilde{F}_{k}(f)\left(y_{\tau}^{k, \nu}\right) E_{k}\left(x, y_{\tau}^{k, \nu}\right) .
\end{aligned}
$$

For all $L \in \mathbb{N}$, by using the definition of the norm $\|\cdot\|_{B_{p q}^{s}(X)}$ and a similar argument to that of Theorem 1 in [4], we can show that 
$(2.21)$

$$
\begin{aligned}
\| f- & f_{L} \|_{B_{p q}^{s}(X)} \\
\leq & C\left\{\sum_{\tau=L+1}^{\infty} \sum_{\nu=1}^{N(0, \tau)} \mu\left(Q_{\tau}^{0, \nu}\right)\left|\widetilde{F}_{Q_{\tau}^{0, \nu}}(f)\right|^{p}\right\}^{1 / p} \\
& +C\left\{\sum_{k=L+1}^{\infty} 2^{k s q}\left(\sum_{\tau \in \mathbb{N}} \sum_{\nu=1}^{N(k, \tau)} \mu\left(Q_{\tau}^{k, \nu}\right)\left[\sup _{z \in Q_{\tau}^{k, \nu}}\left|\widetilde{F}_{k}(f)(z)\right|\right]^{p}\right)^{q / p}\right\}^{1 / q} \\
& +C\left\{\sum_{k=1}^{L} 2^{k s q}\left(\sum_{\tau=L+1}^{\infty} \sum_{\nu=1}^{N(k, \tau)} \mu\left(Q_{\tau}^{k, \nu}\right)\left[\sup _{z \in Q_{\tau}^{k, \nu}}\left|\widetilde{F}_{k}(f)(z)\right|\right]^{p}\right)^{q / p}\right\}^{1 / q} \\
= & H_{1}+H_{2}+H_{3} .
\end{aligned}
$$

Now, since $f \in B_{p q}^{s}(X)$, for any $\delta>0$, by Lemma 5 we can choose $L_{1} \in \mathbb{N}$ such that if $L \geq L_{1}$, then

$$
H_{1}+H_{2}<\delta / 3
$$

For $L>L_{1}$, we write

$$
\begin{aligned}
H_{3} \leq & C\left\{\sum_{k=L_{1}+1}^{\infty} 2^{k s q}\left(\sum_{\tau \in \mathbb{N}} \sum_{\nu=1}^{N(k, \tau)} \mu\left(Q_{\tau}^{k, \nu}\right)\left[\sup _{z \in Q_{\tau}^{k, \nu}}\left|\widetilde{F}_{k}(f)(z)\right|\right]^{p}\right)^{q / p}\right\}^{1 / q} \\
& +C\left\{\sum_{k=1}^{L_{1}} 2^{k s q}\left(\sum_{\tau=L+1}^{\infty} \sum_{\nu=1}^{N(k, \tau)} \mu\left(Q_{\tau}^{k, \nu}\right)\left[\sup _{z \in Q_{\tau}^{k, \nu}}\left|\widetilde{F}_{k}(f)(z)\right|\right]^{p}\right)^{q / p}\right\}^{1 / q} \\
< & \delta / 3+C\left\{\sum_{k=1}^{L_{1}} 2^{k s q}\left(\sum_{\tau=L+1}^{\infty} \sum_{\nu=1}^{N(k, \tau)} \mu\left(Q_{\tau}^{k, \nu}\right)\left[\sup _{z \in Q_{\tau}^{k, \nu}}\left|\widetilde{F}_{k}(f)(z)\right|\right]^{p}\right)^{q / p}\right\}^{1 / q} .
\end{aligned}
$$

Since $f \in B_{p q}^{s}(X)$, by Lemma 5 again, we can choose $L_{2} \geq L_{1}$ such that if $L \geq L_{2}$, then

$$
C\left\{\sum_{k=1}^{L_{1}} 2^{k s q}\left(\sum_{\tau=L+1}^{\infty} \sum_{\nu=1}^{N(k, \tau)} \mu\left(Q_{\tau}^{k, \nu}\right)\left[\sup _{z \in Q_{\tau}^{k, \nu}}\left|\widetilde{F}_{k}(f)(z)\right|\right]^{p}\right)^{q / p}\right\}^{1 / q}<\delta / 3 .
$$

Combining (2.21)-(2.24), we conclude that if $L>L_{2}$, then

$$
\left\|f-f_{L}\right\|_{B_{p q}^{s}(X)}<\delta .
$$

That is, $f_{L}$ converges to $f$ in the norm of $B_{p q}^{s}(X)$ if $\max (p, q)<\infty$. This verifies (i).

To prove (ii), by Lemma 5, we obtain 


$$
\begin{aligned}
\|\lambda\|_{f_{p q}^{s}(X) \leq} & C\left\{\sum_{\tau \in I_{0}} \sum_{\nu=1}^{N(0, \tau)} \mu\left(Q_{\tau}^{0, \nu}\right)\left|\widetilde{E}_{Q_{\tau}^{0, \nu}}(f)\right|^{p}\right\}^{1 / p} \\
& +C\left\|\left\{\sum_{k=1}^{\infty} \sum_{\tau \in I_{k}} \sum_{\nu=1}^{N(k, \tau)}\left[2^{k s}\left|\widetilde{E}_{k}(f)\left(y_{\tau}^{k, \nu}\right)\right| \chi_{Q_{\tau}^{k, \nu}}\right]^{q}\right\}^{1 / q}\right\|_{L^{p}(X)} \\
\leq & C\left\{\sum_{\tau \in I_{0}} \sum_{\nu=1}^{N(0, \tau)} \mu\left(Q_{\tau}^{0, \nu}\right)\left[m_{Q_{\tau}^{0, \nu}}\left(\left|E_{0}(f)\right|\right)\right]^{p}\right\}^{1 / p} \\
& +C\left\|\left\{\sum_{k=1}^{\infty} \sum_{\tau \in I_{k}} \sum_{\nu=1}^{N(k, \tau)}\left[2^{k s} \inf _{z \in Q_{\tau}^{k, \nu}}\left|E_{k}(f)(z)\right| \chi_{Q_{\tau}^{k, \nu}}\right]^{q}\right\}^{1 / q}\right\|_{L^{p}(X)} \\
\leq & C\|f\|_{F_{p q}^{s}(X) \cdot}
\end{aligned}
$$

Thus, (1.8) holds. By Proposition 2.3 in [18] and (i), we can easily see that (2.34) holds in $(\stackrel{\circ}{\mathcal{G}}(\beta, \gamma))^{\prime}$ also in this case. By Lemma 3 and an argument similar to that in Theorem 1 and Lemma 5 , similarly to the case of the spaces $B_{p q}^{s}(X)$, we can show that $(2.20)$ converges in the norm of $F_{p q}^{s}(X)$ when $q<\infty$. This finishes the proof of Theorem 4 .

Proof of Theorem 5. Let $\left\{E_{k}\right\}_{k=0}^{\infty}$ be as in Lemma 2. We first verify (1.10). The following estimates are, respectively, (2.14) and (2.15) in [9]: For $k, k^{\prime} \in \mathbb{Z}_{+}, k \leq k^{\prime}, \tau^{\prime} \in I_{k^{\prime}}$ and $\nu^{\prime}=1, \ldots, N\left(k^{\prime}, \tau^{\prime}\right)$,

$$
\begin{aligned}
& \left|E_{k}\left(u_{Q_{\tau^{\prime}}^{k^{\prime}, \nu^{\prime}}}\right)(x)\right| \\
& \quad \leq C \mu\left(Q_{\tau^{\prime}}^{k^{\prime}, \nu^{\prime}}\right)^{-1 / 2} 2^{-\left(k^{\prime}-k\right)(d+\gamma)} \frac{1}{\left(1+2^{k} \varrho\left(x, y_{\tau^{\prime}}^{k^{\prime}, \nu^{\prime}}\right)\right)^{d+\gamma}},
\end{aligned}
$$

and for $k, k^{\prime} \in \mathbb{Z}_{+}, k \geq k^{\prime}, \tau^{\prime} \in I_{k^{\prime}}$ and $\nu^{\prime}=1, \ldots, N\left(k^{\prime}, \tau^{\prime}\right)$,

$$
\left|E_{k}\left(u_{Q_{\tau^{\prime}}^{k^{\prime}, \nu^{\prime}}}\right)(x)\right| \leq C \mu\left(Q_{\tau^{\prime}}^{k^{\prime}, \nu^{\prime}}\right)^{-1 / 2} 2^{-\left(k-k^{\prime}\right) \beta} \frac{1}{\left(1+2^{k^{\prime}} \varrho\left(x, y_{\tau^{\prime}}^{k^{\prime}, \nu^{\prime}}\right)\right)^{d+\gamma}} .
$$

By (2.25), (2.11) and the Hölder inequality, we have

$$
\begin{aligned}
& \left\{\sum_{\tau \in I_{0}} \sum_{\nu=1}^{N(0, \tau)} \mu\left(Q_{\tau}^{0, \nu}\right)\left[m_{Q_{\tau}^{0, \nu}}\left(\left|E_{0}(f)\right|\right)\right]^{p}\right\}^{1 / p} \\
& \leq C\left\{\sum _ { \tau \in I _ { 0 } } \sum _ { \nu = 1 } ^ { N ( 0 , \tau ) } \mu ( Q _ { \tau } ^ { 0 , \nu } ) \left[\sum_{k^{\prime}=0}^{\infty} \sum_{\tau^{\prime} \in I_{k^{\prime}}} \sum_{\nu^{\prime}=1}^{N\left(k^{\prime}, \tau^{\prime}\right)} \mu\left(Q_{\tau^{\prime}}^{k^{\prime}, \nu^{\prime}}\right)^{-1 / 2} 2^{-k^{\prime}(d+\gamma)}\right.\right. \\
& \left.\left.\quad \times\left|\lambda_{Q_{\tau^{\prime}}^{k^{\prime}, \nu^{\prime}}}\right| \inf _{x \in Q_{\tau}^{0, \nu}} \frac{1}{\left(1+\varrho\left(x, y_{\tau^{\prime}}^{k^{\prime}, \nu^{\prime}}\right)\right)^{d+\gamma}}\right]^{p}\right\}^{1 / p}
\end{aligned}
$$




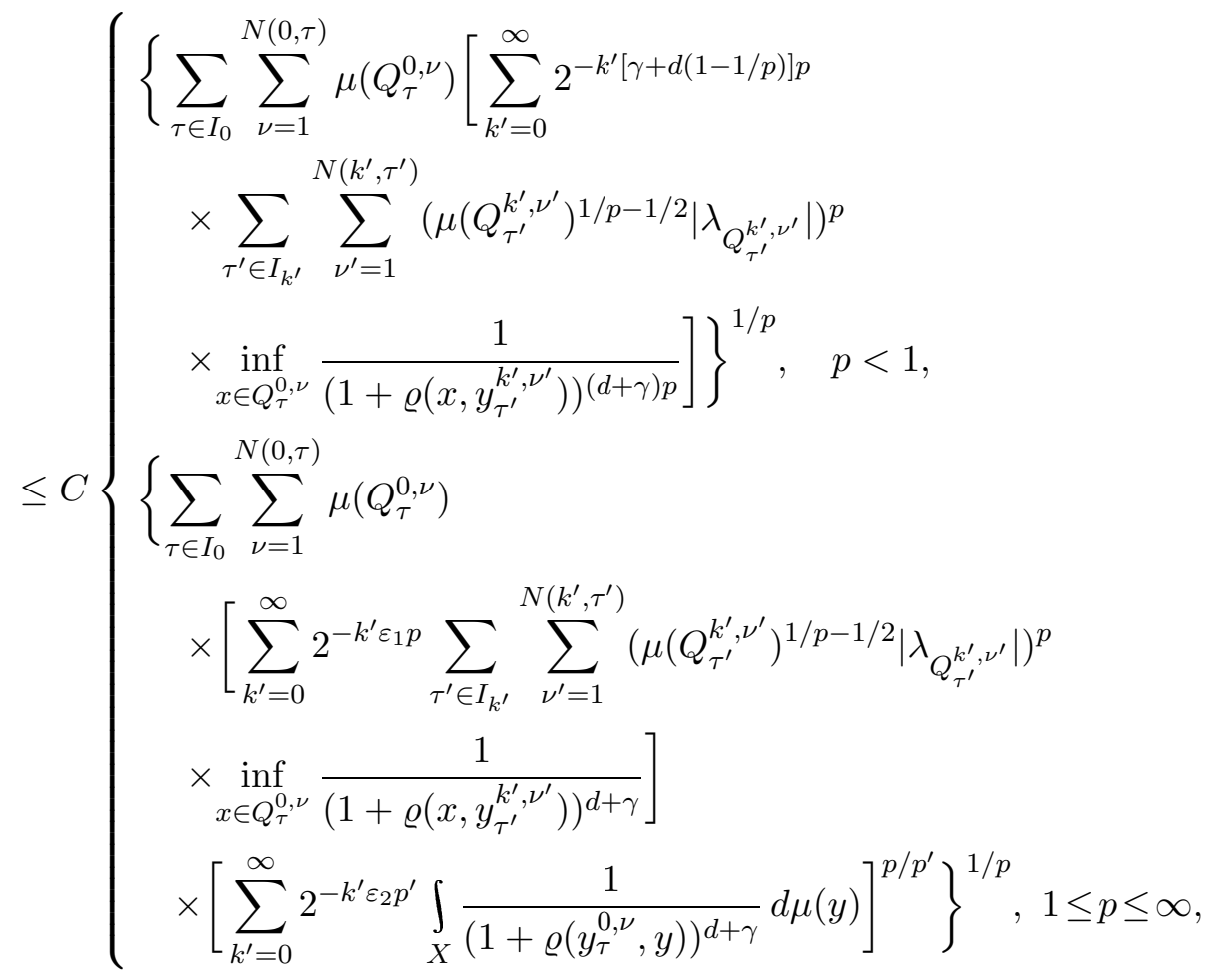

$$
\left\{\begin{array}{l}
\left\{\sum_{k^{\prime}=0}^{\infty} 2^{-k^{\prime}[\gamma+d(1-1 / p)+s] p} 2^{k^{\prime} s p}\right. \\
\left.\quad \times\left[\sum_{\tau^{\prime} \in I_{k^{\prime}}} \sum_{\nu^{\prime}=1}^{N\left(k^{\prime}, \tau^{\prime}\right)}\left(\mu\left(Q_{\tau^{\prime}}^{k^{\prime}, \nu^{\prime}}\right)^{1 / p-1 / 2}\left|\lambda_{Q_{\tau^{\prime}}^{k^{\prime}, \nu^{\prime}}}\right|\right)^{p}\right]\right\}^{1 / p}, p<1, \\
\left\{\sum_{k^{\prime}=0}^{\infty} 2^{-k^{\prime}\left(\varepsilon_{1}+s\right) p} 2^{k^{\prime} s p}\right. \\
\left.\quad \times\left[\sum_{\tau^{\prime} \in I_{k^{\prime}}} \sum_{\nu^{\prime}=1}^{N\left(k^{\prime}, \tau^{\prime}\right)}\left(\mu\left(Q_{\tau^{\prime}}^{k^{\prime}, \nu^{\prime}}\right)^{1 / p-1 / 2}\left|\lambda_{Q_{\tau^{\prime}}^{k^{\prime}, \nu^{\prime}}}\right|\right)^{p}\right]\right\}^{1 / p}, 1 \leq p \leq \infty
\end{array}\right.
$$

$\leq C\|\lambda\|_{b_{p q}^{s}(X)}$,

where we used the fact that

$$
\gamma>\max \left(d(1 / p-1)_{+},-s+d(1 / p-1)_{+}\right)
$$

and we chose $\varepsilon_{1}>0$ and $\varepsilon_{2}>0$ such that $\gamma=\varepsilon_{1}+\varepsilon_{2}$ and $\varepsilon_{1}>-s$.

In what follows, for $k \in \mathbb{N}$, we let 


$$
\begin{aligned}
f_{k} & =\sum_{k^{\prime}=k}^{\infty} \sum_{\tau^{\prime} \in I_{k^{\prime}}} \sum_{\nu^{\prime}=1}^{N\left(k^{\prime}, \tau^{\prime}\right)} \lambda_{Q_{\tau^{\prime}}^{k^{\prime}, \nu^{\prime}}} u_{Q_{\tau^{\prime}}^{k^{\prime}, \nu^{\prime}}}, \\
f^{k} & =\sum_{k^{\prime}=0}^{k-1} \sum_{\tau^{\prime} \in I_{k^{\prime}}} \sum_{\nu^{\prime}=1}^{N\left(k^{\prime}, \tau^{\prime}\right)} \lambda_{Q_{\tau^{\prime}}^{k^{\prime}, \nu^{\prime}}} u_{Q_{\tau^{\prime}}^{k^{\prime}, \nu^{\prime}}}
\end{aligned}
$$

From $(2.25),(2.11)$ and the Hölder inequality, it follows that

$$
\begin{aligned}
& \left\{\sum_{k=1}^{\infty} 2^{k s q}\left(\sum_{\tau \in I_{k}} \sum_{\nu=1}^{N(k, \tau)} \mu\left(Q_{\tau}^{k, \nu}\right)\left[\inf _{x \in Q_{\tau}^{k, \nu}}\left|E_{k}\left(f_{k}\right)(x)\right|\right]^{p}\right)^{q / p}\right\}^{1 / q} \\
& \leq C\left\{\sum _ { k = 1 } ^ { \infty } 2 ^ { k s q } \left(\sum_{\tau \in I_{k}} \sum_{\nu=1}^{N(k, \tau)} \mu\left(Q_{\tau}^{k, \nu}\right)\right.\right. \\
& \times\left[\sum_{k^{\prime}=k}^{\infty} \sum_{\tau^{\prime} \in I_{k^{\prime}}} \sum_{\nu^{\prime}=1}^{N\left(k^{\prime}, \tau^{\prime}\right)} \mu\left(Q_{\tau^{\prime}}^{k^{\prime}, \nu^{\prime}}\right)^{-1 / 2} 2^{-\left(k^{\prime}-k\right)(d+\gamma)}\right. \\
& \left.\left.\left.\times\left|\lambda_{Q_{\tau^{\prime}}^{k^{\prime}, \nu^{\prime}}}\right| \inf _{x \in Q_{\tau}^{k, \nu}} \frac{1}{\left(1+2^{k} \varrho\left(x, y_{\tau^{\prime}}^{k^{\prime}, \nu^{\prime}}\right)\right)^{d+\gamma}}\right]^{p}\right)^{q / p}\right\}^{1 / q} \\
& \left\{\begin{array}{l}
\left\{\sum _ { k = 1 } ^ { \infty } 2 ^ { k ( s + d + \gamma ) q } \left[\sum_{k^{\prime}=k}^{\infty} 2^{-k^{\prime}[\gamma+d(1-1 / p)] p}\right.\right. \\
\quad \times \sum_{\tau^{\prime} \in I_{k^{\prime}}} \sum_{\nu^{\prime}=1}^{N\left(k^{\prime}, \tau^{\prime}\right)}\left(\mu\left(Q_{\tau^{\prime}}^{k^{\prime}, \nu^{\prime}}\right)^{1 / p-1 / 2}\left|\lambda_{Q_{\tau^{\prime}}^{k^{\prime}, \nu^{\prime}}}\right|\right)^{p}
\end{array}\right. \\
& \left.\left.\times \int_{X} \frac{1}{\left(1+2^{k} \varrho\left(x, y_{\tau^{\prime}}^{k^{\prime}, \nu^{\prime}}\right)\right)^{(d+\gamma) p}} d \mu(x)\right]^{q / p}\right\}^{1 / q}, p<1, \\
& \leq C\left\{\begin{aligned}
\{ & \sum_{k=1}^{\infty} 2^{k(s+d+\gamma) q}\left(\sum_{\tau \in I_{k}} \sum_{\nu=1}^{N(k, \tau)} \mu\left(Q_{\tau}^{k, \nu}\right)\right. \\
& \times\left[\sum_{k^{\prime}=k}^{\infty} 2^{-k^{\prime} \varepsilon_{1} p} \sum_{\tau^{\prime} \in I_{k^{\prime}}} \sum_{\nu^{\prime}=1}^{N\left(k^{\prime}, \tau^{\prime}\right)}\left(\mu\left(Q_{\tau^{\prime}}^{k^{\prime}, \nu^{\prime}}\right)^{1 / p-1 / 2}\left|\lambda_{Q_{\tau^{\prime}}^{k^{\prime}, \nu^{\prime}}}\right|\right)^{p}\right.
\end{aligned}\right. \\
& \left.\times \inf _{x \in Q_{\tau}^{k, \nu}} \frac{1}{\left(1+2^{k} \varrho\left(x, y_{\tau^{\prime}}^{k^{\prime}, \nu^{\prime}}\right)\right)^{d+\gamma}}\right] \\
& \left.\left.\times\left[\sum_{k^{\prime}=k}^{\infty} 2^{-k^{\prime} \varepsilon_{2} p^{\prime}} \int_{X} \frac{1}{\left(1+2^{k} \varrho\left(y_{\tau}^{k, \nu}, y\right)\right)^{d+\gamma}} d \mu(y)\right]^{p / p^{\prime}}\right)^{q / p}\right\}^{1 / q},
\end{aligned}
$$




$$
\left\{\begin{array}{l}
\left\{\sum _ { k = 1 } ^ { \infty } \left[\sum_{k^{\prime}=k}^{\infty} 2^{\left(k-k^{\prime}\right)[\gamma+s+d(1-1 / p)] p} 2^{k^{\prime} s p}\right.\right. \\
\left.\left.\quad \times \sum_{\tau^{\prime} \in I_{k^{\prime}}} \sum_{\nu^{\prime}=1}^{N\left(k^{\prime}, \tau^{\prime}\right)}\left(\mu\left(Q_{\tau^{\prime}}^{k^{\prime}, \nu^{\prime}}\right)^{1 / p-1 / 2}\left|\lambda_{Q_{\tau^{\prime}}^{k^{\prime}, \nu^{\prime}}}\right|\right)^{p}\right]^{q / p}\right\}^{1 / q}, \quad p<1, \\
\left\{\sum _ { k = 1 } ^ { \infty } \left[\sum_{k^{\prime}=k}^{\infty} 2^{\left(k-k^{\prime}\right)\left(\varepsilon_{1}+s\right) p} 2^{k^{\prime} s p}\right.\right. \\
\left.\left.\quad \times \sum_{\tau^{\prime} \in I_{k^{\prime}}} \sum_{\nu^{\prime}=1}^{N\left(k^{\prime}, \tau^{\prime}\right)}\left(\mu\left(Q_{\tau^{\prime}}^{k^{\prime}, \nu^{\prime}}\right)^{1 / p-1 / 2}\left|\lambda_{Q_{\tau^{\prime}}^{k^{\prime}, \nu^{\prime}}}\right|\right)^{p}\right]^{q / p}\right\}^{1 / q}, \quad 1 \leq p \leq \infty,
\end{array}\right.
$$

$\leq C\|\lambda\|_{b_{p q}^{s}(X)}$

where we chose $\varepsilon_{1}>0$ and $\varepsilon_{2}>0$ such that $\gamma=\varepsilon_{1}+\varepsilon_{2}$ and $\varepsilon_{1}>-s$, and we used the fact that $\gamma>\max \left(d(1 / p-1)_{+},-s+d(1 / p-1)_{+}\right)$.

By (2.26), (2.11) and the Hölder inequality, we obtain

$$
\left\{\sum_{k=1}^{\infty} 2^{k s q}\left(\sum_{\tau \in I_{k}} \sum_{\nu=1}^{N(k, \tau)} \mu\left(Q_{\tau}^{k, \nu}\right)\left[\inf _{x \in Q_{\tau}^{k, \nu}}\left|E_{k}\left(f^{k}\right)(x)\right|\right]^{p}\right)^{q / p}\right\}^{1 / q}
$$$$
\leq C\left\{\sum _ { k = 1 } ^ { \infty } 2 ^ { k s q } \left(\sum _ { \tau \in I _ { k } } \sum _ { \nu = 1 } ^ { N ( k , \tau ) } \mu ( Q _ { \tau } ^ { k , \nu } ) \left[\sum_{k^{\prime}=0}^{k-1} \sum_{\tau^{\prime} \in I_{k^{\prime}}} \sum_{\nu^{\prime}=1}^{N\left(k^{\prime}, \tau^{\prime}\right)} \mu\left(Q_{\tau^{\prime}}^{k^{\prime}, \nu^{\prime}}\right)^{-1 / 2} 2^{-\left(k-k^{\prime}\right) \beta}\right.\right.\right.
$$$$
\left.\left.\left.\times\left|\lambda_{Q_{\tau^{\prime}}^{k^{\prime}, \nu^{\prime}}}\right| \inf _{x \in Q_{\tau}^{k, \nu}} \frac{1}{\left(1+2^{k^{\prime}} \varrho\left(x, y_{\tau^{\prime}}^{k^{\prime}, \nu^{\prime}}\right)\right)^{d+\gamma}}\right]^{p}\right)^{q / p}\right\}^{1 / q}
$$$$
\int\left\{\sum _ { k = 1 } ^ { \infty } \left[\sum_{k^{\prime}=0}^{k-1} 2^{\left(k-k^{\prime}\right)(s-\beta) p} 2^{k^{\prime} s p}\right.\right.
$$$$
\leq C\left\{\begin{array}{l}
\left.\left.\times \sum_{\tau^{\prime} \in I_{k^{\prime}}} \sum_{\nu^{\prime}=1}^{N\left(k^{\prime}, \tau^{\prime}\right)}\left(\mu\left(Q_{\tau^{\prime}}^{k^{\prime}, \nu^{\prime}}\right)^{1 / p-1 / 2}\left|\lambda_{Q_{\tau^{\prime}}^{k^{\prime}, \nu^{\prime}}}\right|\right)^{p}\right]^{q / p}\right\}^{1 / q}, \quad p<1, \\
\left\{\sum _ { k = 1 } ^ { \infty } \left[\sum_{k^{\prime}=0}^{k-1} 2^{\left(k-k^{\prime}\right)\left(s-\varepsilon_{1}\right) p} 2^{k^{\prime} s p}\right.\right. \\
\left.\left.\quad \times \sum_{\tau^{\prime} \in I_{k^{\prime}}} \sum_{\nu^{\prime}=1}^{N\left(k^{\prime}, \tau^{\prime}\right)}\left(\mu\left(Q_{\tau^{\prime}}^{k^{\prime}, \nu^{\prime}}\right)^{1 / p-1 / 2}\left|\lambda_{Q_{\tau^{\prime}}^{k^{\prime}, \nu^{\prime}}}\right|\right)^{p}\right]^{q / p}\right\}^{1 / q}, \quad 1 \leq p \leq \infty,
\end{array}\right.
$$

$\leq C\|\lambda\|_{b_{p q}^{s}(X)}$ 
where we chose $\varepsilon_{1}>0$ and $\varepsilon_{2}>0$ such that $\beta=\varepsilon_{1}+\varepsilon_{2}$ and $\varepsilon_{1}>s$, and we also used the fact that $\beta>s_{+}$and $\gamma>d(1 / p-1)_{+}$.

Combining (2.27)-(2.29), we obtain (1.10). Using (1.10), we can show that the series in (1.9) converge in the norm of $B_{p q}^{s}(X)$ if $\max (p, q)<\infty$, and in $\left(\stackrel{\circ}{\mathcal{G}}\left(\beta_{1}, \gamma_{1}\right)\right)^{\prime}$ with $\beta_{1}$ and $\gamma_{1}$ as in Theorem 5 , as in the proof of Theorem 3. We leave the details to the reader. This finishes the proof of (i).

Let us now prove (ii). We only verify (1.11). By (2.25), (2.11), Lemma 4 and the Fefferman-Stein vector-valued maximal function inequality in [5], we obtain

$$
\begin{aligned}
& \left\{\sum_{\tau \in I_{0}} \sum_{\nu=1}^{N(0, \tau)} \mu\left(Q_{\tau}^{0, \nu}\right)\left[m_{Q_{\tau}^{0, \nu}}\left(\left|E_{0}(f)\right|\right)\right]^{p}\right\}^{1 / p} \\
& \leq C\left\{\int _ { X } \sum _ { \tau \in I _ { 0 } } \sum _ { \nu = 1 } ^ { N ( 0 , \tau ) } \chi _ { Q _ { \tau } ^ { 0 , \nu } } ( x ) \left[\sum_{k^{\prime}=0}^{\infty} \sum_{\tau^{\prime} \in I_{k^{\prime}}} \sum_{\nu^{\prime}=1}^{N\left(k^{\prime}, \tau^{\prime}\right)} \mu\left(Q_{\tau^{\prime}}^{k^{\prime}, \nu^{\prime}}\right)^{-1 / 2} 2^{-k^{\prime}(d+\gamma)}\right.\right. \\
& \left.\left.\times\left|\lambda_{Q_{\tau^{\prime}}^{k^{\prime}, \nu^{\prime}}}\right| \frac{1}{\left(1+\varrho\left(x, y_{\tau^{\prime}}^{k^{\prime}, \nu^{\prime}}\right)\right)^{d+\gamma}}\right]^{p} d \mu(x)\right\}^{1 / p} \\
& \leq C \| \sum_{k^{\prime}=0}^{\infty} 2^{-k^{\prime}(d+\gamma-d / r+s)} 2^{k^{\prime} s} \\
& \times\left(M\left[\sum_{\tau^{\prime} \in I_{k^{\prime}}} \sum_{\nu^{\prime}=1}^{N\left(k^{\prime}, \tau^{\prime}\right)}\left(\mu\left(Q_{\tau^{\prime}}^{k^{\prime}, \nu^{\prime}}\right)^{-1 / 2}\left|\lambda_{Q_{\tau^{\prime}}^{k^{\prime}, \nu^{\prime}}}\right|\right)^{r} \chi_{Q_{\tau^{\prime}}^{k^{\prime}, \nu^{\prime}}}\right]\right)^{1 / r} \|_{L^{p}(X)} \\
& \leq C \|\left\{\sum_{k^{\prime}=0}^{\infty} 2^{k^{\prime} s q}\right. \\
& \left.\times\left[M\left(\sum_{\tau^{\prime} \in I_{k^{\prime}}} \sum_{\nu^{\prime}=1}^{N\left(k^{\prime}, \tau^{\prime}\right)}\left[\mu\left(Q_{\tau^{\prime}}^{k^{\prime}, \nu^{\prime}}\right)^{-1 / 2}\left|\lambda_{Q_{\tau^{\prime}}^{k^{\prime}, \nu^{\prime}}}\right|\right]^{r} \chi_{Q_{\tau^{\prime}}^{k^{\prime}, \nu^{\prime}}}\right)\right]^{q / r}\right\}^{1 / q} \|_{L^{p}(X)} \\
& \leq C\|\lambda\|_{f_{p q}^{s}(X)},
\end{aligned}
$$

where we chose $r \in(0,1]$ such that $0<r<\min (1, p, q)$ and

$$
\gamma>\max \left(d(1 / r-1)_{+},-s+d(1 / r-1)_{+}\right)
$$

From (2.25), (2.11), Lemma 4 and the Fefferman-Stein vector-valued maximal function inequality in [5], it follows that 


$$
\begin{aligned}
& \left\|\left\{\sum_{k=1}^{\infty} \sum_{\tau \in I_{k}} \sum_{\nu=1}^{N(k, \tau)}\left[2^{k s} \inf _{x \in Q_{\tau}^{k, \nu}}\left|E_{k}\left(f_{k}\right)(x)\right| \chi_{Q_{\tau}^{k, \nu}}\right]^{q}\right\}^{1 / q}\right\|_{L^{p}(X)} \\
& \leq C \|\left\{\sum _ { k = 1 } ^ { \infty } 2 ^ { k s q } \sum _ { \tau \in I _ { k } } \sum _ { \nu = 1 } ^ { N ( k , \tau ) } \left[\sum_{k^{\prime}=k}^{\infty} \sum_{\tau^{\prime} \in I_{k^{\prime}}} \sum_{\nu^{\prime}=1}^{N\left(k^{\prime}, \tau^{\prime}\right)} \mu\left(Q_{\tau^{\prime}}^{k^{\prime}, \nu^{\prime}}\right)^{-1 / 2} 2^{-\left(k^{\prime}-k\right)(d+\gamma)}\right.\right. \\
& \left.\left.\times\left|\lambda_{Q_{\tau^{\prime}}^{k^{\prime}, \nu^{\prime}}}\right| \chi_{Q_{\tau}^{k, \nu}} \frac{1}{\left(1+2^{k} \varrho\left(\cdot, y_{\tau^{\prime}}^{k^{\prime}, \nu^{\prime}}\right)\right)^{d+\gamma}}\right]^{q}\right\}^{1 / q} \|_{L^{p}(X)} \\
& \leq C \|\left\{\sum _ { k = 1 } ^ { \infty } \left[\sum_{k^{\prime}=k}^{\infty} 2^{-\left(k^{\prime}-k\right)(d+\gamma-d / r+s)} 2^{k^{\prime} s}\right.\right. \\
& \left.\left.\times\left(M\left[\sum_{\tau^{\prime} \in I_{k^{\prime}}} \sum_{\nu^{\prime}=1}^{N\left(k^{\prime}, \tau^{\prime}\right)}\left(\mu\left(Q_{\tau^{\prime}}^{k^{\prime}, \nu^{\prime}}\right)^{-1 / 2}\left|\lambda_{Q_{\tau^{\prime}}^{k^{\prime}, \nu^{\prime}}}\right|\right)^{r} \chi_{Q_{\tau^{\prime}}^{k^{\prime}, \nu^{\prime}}}\right]\right)^{1 / r}\right]^{q}\right\}^{1 / q} \|_{L^{p}(X)} \\
& \leq C \|\left\{\sum_{k^{\prime}=1}^{\infty} 2^{k^{\prime} s q}\right. \\
& \left.\times\left[M\left(\sum_{\tau^{\prime} \in I_{k^{\prime}}} \sum_{\nu^{\prime}=1}^{N\left(k^{\prime}, \tau^{\prime}\right)}\left[\mu\left(Q_{\tau^{\prime}}^{k^{\prime}, \nu^{\prime}}\right)^{-1 / 2}\left|\lambda_{Q_{\tau^{\prime}}^{k^{\prime}, \nu^{\prime}}}\right|\right]^{r} \chi_{Q_{\tau^{\prime}}^{k^{\prime}, \nu^{\prime}}}\right)\right]^{q / r}\right\}^{1 / q} \|_{L^{p}(X)} \\
& \leq C\|\lambda\|_{f_{p q}^{s}(X)},
\end{aligned}
$$

where we chose $r \in(0,1]$ as in $(2.30)$; while by $(2.26)$, we also have

$$
\begin{aligned}
& \quad\left\|\left\{\sum_{k=1}^{\infty} \sum_{\tau \in I_{k}} \sum_{\nu=1}^{N(k, \tau)}\left[2^{k s} \inf _{x \in Q_{\tau}^{k, \nu}}\left|E_{k}\left(f^{k}\right)(x)\right| \chi_{Q_{\tau}^{k, \nu}}\right]^{q}\right\}^{1 / q}\right\|_{L^{p}(X)} \\
& \leq C \|\left\{\sum _ { k = 1 } ^ { \infty } 2 ^ { k s q } \sum _ { \tau \in I _ { k } } \sum _ { \nu = 1 } ^ { N ( k , \tau ) } \left[\sum_{k^{\prime}=0}^{k-1} \sum_{\tau^{\prime} \in I_{k^{\prime}}} \sum_{\nu^{\prime}=1}^{N\left(k^{\prime}, \tau^{\prime}\right)} \mu\left(Q_{\tau^{\prime}}^{k^{\prime}, \nu^{\prime}}\right)^{-1 / 2} 2^{-\left(k-k^{\prime}\right) \beta}\right.\right. \\
& \left.\left.\times\left|\lambda_{Q_{\tau^{\prime}}^{k^{\prime}, \nu^{\prime}}}\right| \chi_{Q_{\tau}^{k, \nu}} \frac{1}{\left(1+2^{k^{\prime}} \varrho\left(\cdot, y_{\tau^{\prime}}^{k^{\prime}, \nu^{\prime}}\right)\right)^{d+\gamma}}\right]^{1 / q}\right\}_{L^{p}(X)}\left\|_{\leq}\right\|\left\{\sum _ { k = 1 } ^ { \infty } \left[\sum_{k^{\prime}=0}^{k-1} 2^{-\left(k-k^{\prime}\right)(\beta-s)} 2^{k^{\prime} s}\right.\right. \\
& \left.\left.\quad \times\left(M\left[\sum_{\tau^{\prime} \in I_{k^{\prime}}} \sum_{\nu^{\prime}=1}^{N\left(k^{\prime}, \tau^{\prime}\right)}\left(\mu\left(Q_{\tau^{\prime}}^{k^{\prime}, \nu^{\prime}}\right)^{-1 / 2}\left|\lambda_{Q_{\tau^{\prime}}^{k^{\prime}, \nu^{\prime}}}\right|\right)^{r} \chi_{Q_{\tau^{\prime}}^{k^{\prime}, \nu^{\prime}}}\right]\right)^{1 / r}\right]^{q}\right\}^{1 / q} \|_{L^{p}(X)}
\end{aligned}
$$




$$
\begin{aligned}
& \leq C \|\left\{\sum_{k^{\prime}=0}^{\infty} 2^{k^{\prime} s q}\right. \\
& \left.\quad \times\left[M\left(\sum_{\tau^{\prime} \in I_{k^{\prime}}} \sum_{\nu^{\prime}=1}^{N\left(k^{\prime}, \tau^{\prime}\right)}\left[\mu\left(Q_{\tau^{\prime}}^{k^{\prime}, \nu^{\prime}}\right)^{-1 / 2}\left|\lambda_{Q_{\tau^{\prime}}^{k^{\prime}, \nu^{\prime}}}\right|\right]^{r} \chi_{Q_{\tau^{\prime}}^{k^{\prime}, \nu^{\prime}}}\right)\right]^{q / r}\right\}^{1 / q} \|_{L^{p}(X)} \\
& \leq C\|\lambda\|_{f_{p q}^{s}(X),}
\end{aligned}
$$

where we chose again $r \in(0,1)$ as in (2.30) and we also used the fact that $\beta>s_{+}$. Now, (2.30)-(2.32) yield (1.11).

This finishes the proof of Theorem 5 .

3. Some remarks. First, we point out that on $\mathbb{R}^{n},(1.3)$ and (1.4) with a special choice of $E_{k}$ are true even when $p<1$. In fact, in this case, we can take $E_{0}(f)(x)=(\phi * f)(x)$ and $E_{k}(f)(x)=\left(\psi_{k} * f\right)(x)$ for $k \in \mathbb{N}$, where $\phi$ and $\psi$ are Schwartz functions, supp $\widehat{\phi} \subset\left\{x \in \mathbb{R}^{n}:|x| \leq 2\right\},|\widehat{\phi}(x)| \geq C>0$ for all $|x| \leq 1$,

$$
\operatorname{supp} \widehat{\psi}(x) \subset\left\{x \in \mathbb{R}^{n}: 1 / 2 \leq|x| \leq 2\right\},
$$

$|\widehat{\psi}(x)| \geq C>0$ for all $3 / 5 \leq|x| \leq 5 / 3$, and $\psi_{k}(x)=\psi\left(2^{-k} x\right)$ for $k \in \mathbb{N}$ (see [15]). Let $\mathcal{D}$ be the set of all dyadic cubes with side length $2^{-j}$. By the following well-known Plancherel-Pólya inequality on $\mathbb{R}^{n}$ :

$$
\sum_{Q \in \mathcal{D}}|Q| \sup _{z \in Q}|(\phi * f)(z)|^{p} \leq C\|\phi * f\|_{L^{p}(X)}^{p}
$$

for all $p \in(0, \infty)$ (see $[15])$, we can deduce

$$
\begin{aligned}
& \sum_{Q \in \mathcal{D}}|Q|\left[\frac{1}{|Q|} \int_{Q} \mid(\phi * f)\right.(x) \mid d \mu(x)]^{p} \\
& \leq C \sum_{Q \in \mathcal{D}}|Q| \sup _{z \in Q}|(\phi * f)(z)|^{p} \leq C\|\phi * f\|_{L^{p}(X)}^{p}
\end{aligned}
$$

From this and other inequalities in the proof of Theorem 2, it follows that (1.3) and (1.4) are also true on $\mathbb{R}^{n}$ when $p, q<1$ with these special operators. We also remark that if $s \in(0, \theta)$ and $u_{Q_{\tau}^{k, \nu}}$ for all $k \in \mathbb{Z}_{+}, \tau \in I_{k}$ and $\nu=1, \ldots, N(k, \tau)$ are smooth units, then Theorem 5 still holds, which can be seen from its proof. From this fact and Theorem 6.6 of [19], we can see that if $X$ is a Lipschitz manifold, then the spaces $B_{p q}^{s}(X)$ and $F_{p q}^{s}(X)$ are the same as those defined in [19] by a completely different approach; see [19] for the details.

Finally, we remark that (1.5) can be proved without using Theorems 4 and 5 of this paper and Theorems 2.1 and 2.2 of [10]. One can prove (1.5) by applying Lemma 4, Theorem 2.1 of [10], the Fefferman-Stein vector-valued 
maximal function inequality of [5] and the definition of the spaces $F_{p q}^{s}(X)$ in terms of the Littlewood-Paley $S$-function; see also [4] and [7].

Acknowledgments. Dachun Yang sincerely thanks Professor Hans Triebel for many helpful discussions on this subject, especially for his idea on distribution spaces.

Both authors are greatly indebted to the referee for several helpful suggestions which led to Remark 2, and to the editor for his careful reading and valuable remarks which made this article more readable.

\section{References}

[1] M. Christ, $A T(b)$ theorem with remarks on analytic capacity and the Cauchy integral, Colloq. Math. 60/61 (1990), 601-628.

[2] R. R. Coifman et G. Weiss, Analyse Harmonique Non-commutative sur Certains Espaces Homogènes, Lecture Notes in Math. 242, Springer, Berlin, 1971.

[3] G. David, J.-L. Journé et S. Semmes, Opérateurs de Calderón-Zygmund, fonctions para-accrétives et interpolation, Rev. Mat. Iberoamericana 1 (1985), 1-56.

[4] D. Deng, Y. S. Han and D. C. Yang, Inhomogeneous Plancherel-Pólya inequalities on spaces of homogeneous type and their applications, submitted.

[5] C. Fefferman and E. M. Stein, Some maximal inequalities, Amer. J. Math. 93 (1971), $107-116$.

[6] M. Frazier and B. Jawerth, A discrete transform and decompositions of distribution spaces, J. Funct. Anal. 93 (1990), 34-170.

[7] Y. S. Han, Plancherel-Pólya type inequality on spaces of homogeneous type and its applications, Proc. Amer. Math. Soc. 126 (1998), 3315-3327.

[8] - Inhomogeneous Calderón reproducing formula on spaces of homogeneous type, J. Geom. Anal. 7 (1997), 259-284.

[9] Y. S. Han, S. Lu and D. C. Yang, Inhomogeneous Besov and Triebel-Lizorkin spaces on spaces of homogeneous type, Approx. Theory Appl. 15 (1999), no. 3, 37-65.

[10] - - - - Inhomogeneous Triebel-Lizorkin spaces on spaces of homogeneous type, Math. Sci. Res. Hot-Line 3 (1999), no. 9, 1-29.

[11] —, - - - Inhomogeneous discrete Calderón reproducing formulas for spaces of homogeneous type, J. Fourier Anal. Appl. 7 (2001), 571-600.

[12] Y. S. Han and E. T. Sawyer, Littlewood-Paley theory on spaces of homogeneous type and classical function spaces, Mem. Amer. Math. Soc. 530 (1994).

[13] Y. S. Han and D. C. Yang, New characterizations and applications of inhomogeneous Besov and Triebel-Lizorkin spaces on homogeneous type spaces and fractals, Dissertationes Math. (Rozprawy Mat.) 403 (2002).

[14] R. A. Macías and C. Segovia, Lipschitz functions on spaces of homogeneous type, Adv. Math. 33 (1979), 257-270.

[15] H. Triebel, Theory of Function Spaces, Birkhäuser, Basel, 1983.

[16] —, Theory of Function Spaces II, Birkhäuser, Basel, 1992.

[17] —, Fractals and Spectra, Birkhäuser, Basel, 1997.

[18] —, The Structure of Functions, Birkhäuser, Basel, 2001.

[19] - Function spaces in Lipschitz domains and on Lipschitz manifolds. Characteristic functions as pointwise multipliers, Rev. Mat. Complut. 15 (2002), 1-50. 
[20] H. Triebel and D. C. Yang, Spectral theory of Riesz potentials on quasi-metric spaces, Math. Nachr. 238 (2002), 160-184.

[21] D. C. Yang, Frame characterizations of Besov and Triebel-Lizorkin spaces on spaces of homogeneous type and their applications, Georgian Math. J. 9 (2002), 567-590.

Department of Mathematics

Auburn University

Auburn, AL 36849-5310, U.S.A.

E-mail: hanyong@mail.auburn.edu
Department of Mathematics Beijing Normal University Beijing 100875

People's Republic of China E-mail: dcyang@bnu.edu.cn

Received May 13, 2002

Revised version September 16, 2002 